\title{
Exploiting Impacts of Antenna Selection and Energy Harvesting for Massive Network Connectivity
}

\author{
Minh-Sang Van Nguyen, Dinh-Thuan Do, Senior Member, IEEE, Saba Al-Rubaye, Senior Member, IEEE, Shahid \\ Mumtaz, Senior Member, IEEE, Anwer Al-Dulaimi, Senior Member, IEEE, and Octavia Dobre, Fellow, IEEE
}

\begin{abstract}
As a new energy saving approach for green communications, energy harvesting $(\mathrm{EH})$ could be suitable technique to facilitate massive connections for large number of devices in such networks. The spectrum shortage occurs in huge number of devices which access with small-cell and macro-cell networks. To tackle these challenges, we develop a tractable framework relying on prominent techniques such as non-orthogonal multiple access (NOMA), antenna selection and energy harvesting. In this paper, we aim at practical scenarios of small cell networks by jointly evaluating capable of interference management and EH. We benefit from transmission approaches including full duplex (FD) and bi-directional transmission to improve the main performance system metrics such as outage probability and throughput. Three useful schemes are explored by considering EH and inter-cell interference. We derive the closed-form and asymptotic expressions for system metrics. We then perform extensive simulations with different system configurations to confirm the effectiveness of the proposed small-cell NOMA systems.
\end{abstract}

Index Terms-Small-cell, transmit antenna selection, outage probability, non-orthogonal multiple access.

\section{INTRODUCTION}

V Arious kinds of orthogonal multiple access (OMA) schemes are usually employed in current wireless communication systems, and these techniques are classified following time division, frequency division, and code division for multiple access function. In such schemes, to avoid possible multi-user interference multiple users are served, in which one mobile user (MU) is entirely assigned by one resource block. In practice, although the cost of low spectral efficiency, the OMA schemes are relatively easy to deploy. Benefiting from the superior performance of spectrum utilization, NOMA has attracted attentions due to advantages of massive connectivity and low latency $[1,2,3]$. Different from the traditional OMA, power is high popularity in research compared with timebased or frequency domain-based NOMA schemes $[4,5]$. To enabling domain-based NOMA scheme, different power levels

M.S. Van Nguyen is with the Faculty of Electronics Technology, Industrial University of Ho Chi Minh City (IUH), Ho Chi Minh City, Viet Nam (email: nguyenvanminhsang@iuh.edu.vn).

D.T. Do (Corresponding author) is with the Department of Electrical and Computer Engineering, The University of Texas at Austin, Austin, TX 78712, USA (email: dodinhthuan@utexas.edu).

S. Al-Rubaye, School of Aerospace, Transport and Manufacturing, Cranfield University, Bedford MK43 0AL, United Kingdom (e-mail: s.alrubaye@cranfield.ac.uk).

S. Mumtaz is with Instituto de Telecomunicações, Aveiro, Portugal (email: smumtaz@av.it.pt).

A. Al-Dulaimi is with R\&D, EXFO, Montreal, QC H4S 0A4, Canada (email: anwer.al-dulaimi@exfo.com).

O. Dobre is with Engineering and Applied Science, Memorial University, NL A1C 5S7, Canada (email: odobre@mun.ca). are allocated for group of users in NOMA networks and such allocation strategy depends on their channels or requirements, and then their signals are superposed at transmit side over the same channel [6]. At receiver side, to eliminate the co-channel interference, successive interference cancellation (SIC) is implemented and the expected message can be extracted from the received signals $[7,8]$.

\section{A. Related Work and Motivation}

Small-cell networks in heterogeneous networks (HetNets) have been researched to achieve improvements in terms of the ubiquitous service and the system capacity. However, the deployment of massive terminals in numerous small-cell meets several intractable challenges in terms of the energy consumption. The dense implementation of small networks for $5 \mathrm{G}$ and beyond networks pose new further challenges regarding energy-efficient network management and spectrum efficiency.

By considering the applications of NOMA in HetNet, Liu et al. [9] proposed a hybrid HetNet, where macro cells need massive multiple-input-multiple-output (MIMO) transmission while small cells implement NOMA transmission. It could be interesting result as conducting NOMA in HetNet provides better performance in comparison with the traditional OMAbased HetNet. Liu et al. [10] proposed non-cooperative and cooperative schemes to evaluate coverage performance of the HetNet in downlink, and they indicated that coverage improvement of all NOMA users is resulted from design of cooperative NOMA in the situation each NOMA user needs proper power allocations. The spectrum-efficiency improvement in NOMA is also beneficial to small/macro cells since multiple MUs' offloading operating the same channel [11]. The authors in [12] derived the average successful transmission probability by implementing analysis from stochastic geometry, and this model together with opportunistic NOMA transmissions are employed in small cell networks. The subchannel allocation and power allocation are performed to achieve maximal energy efficiency (EE) for the a NOMA HetNet system containing small cells and macro-cell [13]. They considered a mixed integer non-convex optimization problem by determining the cross-tier interference and co-channel interference, and then they studied the problem of energy efficient resource allocation [13]. Different channel gains to users in small-cell network using NOMA with SIC due to the difference location of base stations (BSs), and hence, pairing the right BS with a MU is important [14]. They concerned problem of controlling its transmit power in the uplink. 
Design of multiple antennas at the BS benefits to wireless network even if MUs fabricated with a single antenna. Both the $\mathrm{BS}$ and the MUs are equipped multiple-antenna to introduce more general architecture as in [15]. In particular, a signal alignment scheme was proposed to mitigate interference existing in both the intra-cluster and inter-cluster. Furthermore, multiple input multiple output system has been designed in new architecture as combining NOMA scheme herein, namely MIMO-NOMA, to benefit the spatial degrees of freedom $[16,17,18,19,20]$. For reduced cost of deployment of NOMA-based system, the antenna selection (AS) technique has been introduced as an effective scheme to avoid the high hardware costs, demanding power consumption and heavy computational while maintaining benefits from MIMO in terms of the diversity and throughput [21, 22, 23]. There are only a few papers that considered the AS problem for MIMONOMA systems in the open literature. Specifically, advantages of a transmit AS (TAS) algorithm reported in [24, 25] should be included in NOMA networks together with their analytical characterizations of the system performance.

Besides, EE in wireless networks containing energy limited devices has attracted increasing attention [26]. Two different directions of researches can be investigated with respect to improve energy-efficient. The first group of previous paper considered on finding optimal EE [27]. In [28], system performance in a NOMA system is optimized in term of an EE power allocation strategy and they introduced a low-complexity suboptimal scheme including power allocation and subchannel assignment. The second direction lies in EH. Recently, to enhance of the wireless communication networks in term of the EE, RF signals have been evaluated as viable new sources to implement $\mathrm{EH}$ as recent works in [29, 30, 31, 32, 33, 34]. Motivated by these approaches, we design linear EH in the perspective of HetNet manner.

Spectrum efficiency, EE, and outage probability could be considered three important characteristics of each HetNet system where the trade-off among them should be investigated. Recently, it is predicted that future HetNets should be able to provide massive connectivity to adapt to the fast improvement of mobile internet and proliferation of mobile devices. It is worth pointing out that such networks require an extremely provocative task for the OMA schemes due to limited radio resources. On the other hand, cell-edge users need to replenish to retain their operations. To address these challenges, by considering the energy harvesting approach, we propose different problem formulations based on advances of NOMA strategy that maximize benefits as concerned. Although the higher number of antennas provides the higher system capacity and the higher amount of harvested energy, but these advantages require at higher cost of radio-frequency (RF) chains, computational complexity, and high power consumption such higher costs happen at both the transmitter and receivers in term of signal processing. Therefore, TAS is adopted to relax cost of hardware design. To the best of our knowledge, this is the first work which investigates the NOMA based HetNet with considering energy harvesting and TAS architecture.

\section{B. Main Contributions}

Although the authors in [35] indicated the benefits of NOMA incorporated in small-cell networks with hybrid automatic repeat request (HARQ), energy shortage needs be considered. Therefore, our study focus on $\mathrm{EH}$ to wireless charge for large number of small devices. The similar work considered in [36] regarding a downlink NOMA to implement HetNet. In their system model, the macro BS (MBS) enables wireless backhaul to co-work with multiple small cell BSs (SBSs). The NOMA users can be grouped associated with small cell and the corresponding SBS [36]. From the system explored in [36], we can observe that achieved EE depends on the number of SBSs per MBS. However, the system performance relies on the effect of severe interference when MBS co-works with too many small cells. This motivated us to address such impact of interference. Reference [37] proposed mmWave-NOMA transmission for a machine-tomachine (M2M) network under the context of HetNet. In such, the same resource block can be shared among smallcell users (SCUs) and machine type communication (MTC) devices. In the mentioned work [37], only NOMA user pairs communicate with the SBSs. Further capable of bi-directional transmission among two NOMA user pairs was not well studied. In contrast, our paper examines benefit of bi-direction transmission mode, linear EH in the downlink small-cell to serve better performance for user pairs.

The main contributions are summarized as follows:

1) We consider the NOMA to support bi-direction transmission in the context of a HetNet. The NOMA technique allows the multiple antenna-assisted BS to serve users at different power level allocated. To the authors' best knowledge, this is the first work that considers performance of typical scenarios in small-cell NOMA.

2) Due to the dense unplanned deployments of small cells, loud neighbors, and the closed subscriber group access, HetNet often meets worse performance since inter-interference. Therefore, this paper examines three scenarios related ability of $\mathrm{EH}$, interference between macro-cell users (MCUs) and SCUs. Under impact of interference, a general framework in this paper is necessary to evaluate performance metrics.

3) We derive the closed-form expressions of the outage probability. Under different designs of proposed schemes, we optimally allocate the power level to each user and percentage of harvested power such that the outage probabilities are minimized. In addition, comparing three schemes in this paper provides guidelines as joint deployments of linear EH, small-cell, NOMA schemes.

The rest of this paper is organized as follows. Section II describes the NOMA to support bi-directional transmission. In Section III, we consider the scenario of downlink NOMA in which only NOMA users communicate without impact of interference from normal cellular users and analyze the outage performance. In Section IV present Scheme 2 for case of degradation performance at NOMA users under interference impact from the normal cellular user. While $\mathrm{EH}$ is further 
TABLE I: Comparison of the proposed scheme with similar ideas.

\begin{tabular}{|l|c|c|c|c|c|c|c|c|}
\hline & Our Scheme & {$[3]$} & {$[7]$} & {$[8]$} & {$[37]$} & {$[39]$} & {$[40]$} & {$[43]$} \\
\hline Best AS at SBS & $\mathrm{x}$ & $\mathrm{x}$ & & & & & & \\
\hline Full-Duplex & $\mathrm{x}$ & & & & & & & \\
\hline Bi-Directional signal & $\mathrm{x}$ & & & & & & $\mathrm{x}$ & \\
\hline Impact of interference & $\mathrm{x}$ & & $\mathrm{x}$ & $\mathrm{x}$ & $\mathrm{x}$ & $\mathrm{x}$ & & \\
\hline EH-assisted users & $\mathrm{x}$ & & & & & & & $\mathrm{x}$ \\
\hline Outage probability & $\mathrm{x}$ & $\mathrm{x}$ & $\mathrm{x}$ & & $\mathrm{x}$ & & $\mathrm{x}$ & $\mathrm{x}$ \\
\hline
\end{tabular}

provided to remain operation of NOMA users in case of limited-power at such users and Scheme 3 describes in section $\mathrm{V}$ for such situation. We conduct extensive simulations in Section VI, and Section VII concludes the paper.

\section{System Model}

Consider cellular downlink communications in small-cell network in which SBS transmits superimposed signals to two full-duplex NOMA users simultaneously, shown in Fig. 2(a), Fig. 2(b) and Fig. 2(c). In this scenario, two users can communicate to each other in Scheme 1. To look on impact of interference from the MCU on both end user 1 and user 2, we introduce a framework as Scheme 2. In addition, EH approach benefits to the links between the MCU and two NOMA users, which case is presented in Scheme 3. The SBS is equipped $K$ antennas while MCU and two SCUs are with single antenna ${ }^{1}$. We need two phases to proceed a signal frame, each phase is $T / 2$. The main parameters and functions can be found in the Table II.

Initially, the two users' superposed information is proceeded to serve users, i.e. User 1 and User 2 are denoted by $D_{1}$ and $D_{2}$, respectively. Which are in degraded performance with and without interference from macro cells. In the second phase related to bi-directional connection, $D_{1}$ transmits information to $D_{2}$ and vice versa. Two users $D_{i},(i=1,2)$ are capable of $\mathrm{EH}$ or not.

\section{Scheme 1: Separate Small-Cell}

The signal received by user $D_{i},(i=1,2)$ is given by

$$
y_{D i}^{s 1}=g_{i, k}\left(\sqrt{a_{1} P_{S}} x_{1}+\sqrt{a_{2} P_{S}} x_{2}\right)+w_{i},
$$

where satisfying $a_{1}+a_{2}=1$. We assume that user $D_{1}$ is located at far distance compared with another, and hence higher potion of power needs be assigned to user $D_{1}$, i.e. condition $a_{1}>a_{2}$ must be guaranteed. Further, those factors are determined by the quality of the channel coefficients. It is further assumed a normalized unit power at the SBS, $\mathrm{E}\left\{\left|x_{i}\right|^{2}\right\}=1$. In this scenario, users $D_{1}$ and $D_{2}$ are the non-SIC user and SIC user, respectively.

Then, the received signal to interference plus noise ratio (SINRs) at user $D_{1}$ can be formulated as

$$
\gamma_{1, k}^{s 1}=\frac{a_{1} \rho\left|g_{1, k}\right|^{2}}{a_{2} \rho\left|g_{1, k}\right|^{2}+1}
$$

${ }^{1}$ We study similar scenario of the downlink transmission in a two-tier network as reported in [36]. Such system model in [36] contains one MBS in the macro-cell tier and some SBSs. However, performance of several SBSs are similar, and thus we focus on performance analysis for a SBS.

\begin{tabular}{|c|c|}
\hline Notations & Meaning \\
\hline $\operatorname{Pr}(),. \mathrm{E}\{\}$. & Probability operator, Expectation operator \\
\hline$f_{X}(),. F_{X}()$. & $\begin{array}{l}\text { The probability distribution function (PDF), Cumulative } \\
\text { distribution function (CDF) }\end{array}$ \\
\hline$C N \sim(a, b)$ & $\begin{array}{l}\text { Complex normal distribution with mean } a \text { and variance } \\
b\end{array}$ \\
\hline$a_{i}, d_{i}, \alpha$ & $\begin{array}{l}\text { The power allocation factors to } i \text {-th user, normalized } \\
\text { distances from SBS to } D_{i} \text {, path loss exponent }\end{array}$ \\
\hline$\eta, \beta$ & $\begin{array}{l}\text { The energy conversion coefficient with } 0<\eta<1 \text {, the } \\
\text { power splitting ratio } 0<\beta<1[41,42]\end{array}$ \\
\hline$T, K, \varpi$ & $\begin{array}{l}\text { The whole signal frame, number of transmission anten- } \\
\text { nas, conversion operation betwen FD/half-duplex(HD) } \\
\text { each other }\end{array}$ \\
\hline$P_{S}, P_{D i}, P_{R}$ & $\begin{array}{l}\text { The normalized transmission powers at the SBS, at the } \\
D_{i},(i=1,2) \text {, transmit power from MCU to } D_{i}\end{array}$ \\
\hline$w_{i}$ & $\begin{array}{l}\text { Noise for user } D_{i} \text {, i.e. } w_{i} \sim C N\left(0, N_{0}\right) \text { with } N_{0} \text { is } \\
\text { the normalized noise variance }\end{array}$ \\
\hline$\rho$ & $\begin{array}{l}\text { The transmit signal-to-noise radio (SNR) with } P / N_{0} \text {, } \\
P=P_{S}=P_{D i}=P_{R}\end{array}$ \\
\hline$x_{i}, x_{F D I}, x_{R}$ & $\begin{array}{l}\text { Transmit symbols for user } D_{i} \text {, loop interference signal } \\
\text { at the } D_{i} \text {, signal transmitting from MCU to } D_{i}\end{array}$ \\
\hline$\overline{R_{i}}$ & The target data rate of $D_{i}$ \\
\hline$g_{i, k},(i=1,2)$ & $\begin{array}{l}\text { The Rayleigh fading channel coefficients of correspond- } \\
\text { ing links SBS- } D_{i} \text { with average power of } \lambda_{i}=d_{i}{ }^{\theta}\end{array}$ \\
\hline$l_{i},(i=1,2)$ & $\begin{array}{l}\text { The Rayleigh fading channel coefficients of correspond- } \\
\text { ing links MCU- } D_{i} \text { with average power of } \lambda_{l_{i}}\end{array}$ \\
\hline$h_{1}$ & $\begin{array}{l}\text { The Rayleigh fading channel coefficients of correspond- } \\
\text { ing links } D_{2}-D_{1} \text { with average power of } \lambda_{h_{1}}\end{array}$ \\
\hline$h_{2}$ & $\begin{array}{l}\text { The Rayleigh fading channel coefficients of correspond- } \\
\text { ing links } D_{1}-D_{2} \text { with average power of } \lambda_{h_{2}}\end{array}$ \\
\hline$f_{1}$ & $\begin{array}{l}\text { The Rayleigh fading channel coefficients of correspond- } \\
\text { ing links } D_{1}-D_{1} \text { with average power of } \lambda_{f_{1}}\end{array}$ \\
\hline$f_{2}$ & $\begin{array}{l}\text { The Rayleigh fading channel coefficients of correspond- } \\
\text { ing links } D_{2}-D_{2} \text { with average power of } \lambda_{f_{2}}\end{array}$ \\
\hline
\end{tabular}

TABLE II: Definitions of Notations.

In term of SIC user, the SINR to detect $x_{1}$ and the received SNR at user $D_{2}$ is given by

$$
\gamma_{1 \leftarrow 2, k}^{s 1}=\frac{a_{1} \rho\left|g_{2, k}\right|^{2}}{a_{2} \rho\left|g_{2, k}\right|^{2}+1}, \gamma_{2, k}^{s 1}=a_{2} \rho\left|g_{2, k}\right|^{2} .
$$

In this phase, the user with a stronger channel gain and the user with a weaker gain need cooperate. Designing bidirectional link can assist user $D_{1}$ to decode its data, or user $D_{2}$ to operate SIC better. In such situation, received signal obtained by user $D_{i}$ is expressed as

$$
z_{D i}^{s 1}=\sqrt{P_{D i}} h_{i} x_{i}+\sqrt{\varpi P_{D i}} f_{i} x_{F D I}+w_{i},
$$

where $\varpi=1$ denotes user $D_{i}$ working in FD.

Regarding condition to exist such recieved signal, when $\left|g_{1, k}\right|^{2}>\left|g_{2, k}\right|^{2}$, only $z_{D 2}^{s 1}$ happens, and when $\left|g_{1, k}\right|^{2}<$ $\left|g_{2, k}\right|^{2}$, only $z_{D 1}^{s 1}$ is transmitted from user $D_{2}$. Then, the received SINR at user $D_{i}$ is given by

$$
\chi_{i}^{s 1}=\frac{\rho\left|h_{i}\right|^{2}}{\varpi \rho\left|f_{i}\right|^{2}+1} .
$$




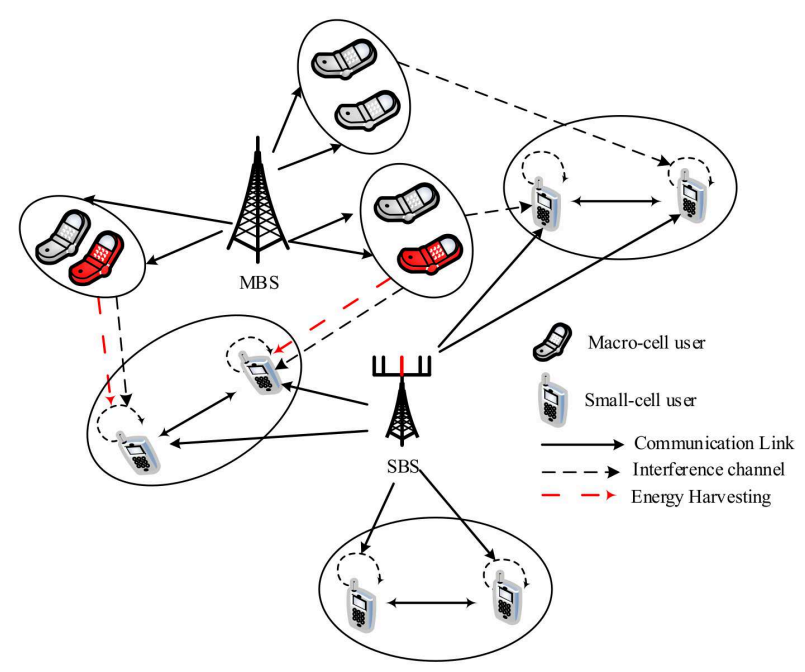

Fig. 1: The system model of small-cell NOMA

Here, if a certain user receives the cooperation signal, the direct transmission and bi-directional transmission joint provide the better of the signals received. The SINR for decoding $x_{1}$ given by

$$
\wp_{D 1}^{s 1}=\left\{\begin{array}{l}
\min \left\{\max \left\{\gamma_{1, k}^{s 1}, \chi_{1}^{s 1}\right\}, \gamma_{1 \leftarrow 2, k}^{s 1}\right\}, \text { if }\left|g_{1, k}\right|^{2}<\left|g_{2, k}\right|^{2} \\
\min \left\{\gamma_{1, k}^{s 1}, \max \left\{\gamma_{1 \leftarrow 2, k}^{s 1}, \chi_{2}^{s 1}\right\}\right\}, \text { otherwise. }
\end{array}\right.
$$

Since only $x_{1}$ is shared for cooperation, the SINR for decoding $x_{2}$ is $\wp_{D 2}^{s 1}=\gamma_{2, k}^{s 1}$ and the data rate of $x_{i}$ in bi-directional cooperative NOMA becomes $R_{D i}^{s 1}=\log _{2}\left(1+\wp_{D 2}^{s 1}\right)$.

The antenna element can be selected to strengthen the link between the SBS and user $D_{i}$ as follow $k^{*}=$ $\arg \underbrace{\max }_{k=1, \ldots, K}\left(\left|g_{i, k}\right|^{2}\right),(i=1,2)$ [38].

The selected channel has CDF, and PDF of $\left|g_{i, k^{*}}\right|^{2}$ respectively as [39]

$$
\begin{gathered}
F_{\left|g_{i, k^{*}}\right|^{2}}(x)=1-\sum_{k=1}^{K}\left(\begin{array}{l}
K \\
k
\end{array}\right)(-1)^{k-1} \exp \left(-\frac{k x}{\lambda_{i}}\right), \\
f_{\left|g_{i, k^{*}}\right|^{2}}(x)=\sum_{k=1}^{K}\left(\begin{array}{l}
K \\
k
\end{array}\right)(-1)^{k-1} \frac{k}{\lambda_{i}} \exp \left(-\frac{k x}{\lambda_{i}}\right) .
\end{gathered}
$$

\section{A. Outage probability of user 1 in Scheme 1 using FD mode}

We evaluate system performance under condition of the predefined data rates $R_{D 1}^{s 1}$ and $R_{D 2}^{s 1}$, and they are determined by the users' QoS requirements. More importantly, the outage probability is considered as an important performance criterion. Depending ability of decode processing, condition of outage behavior happens based on capability of $\gamma_{1 \leftarrow 2, k^{*}}^{s 1}$ compared with condition of required rates. It is noted that outage event occurs at the non-SIC user or the SIC user regarding using the cooperation signal from bidirectional link.

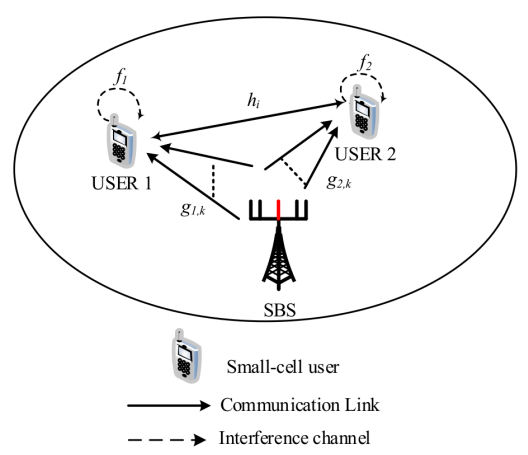

(a) Without interference from $\mathrm{MCU}$

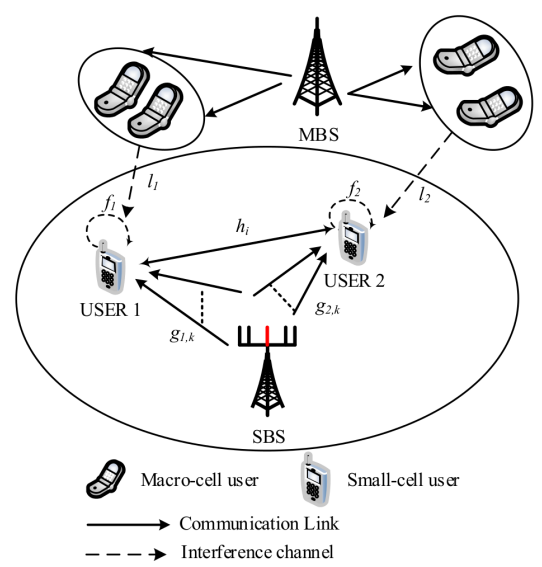

(b) With interference from $\mathrm{MCU}$

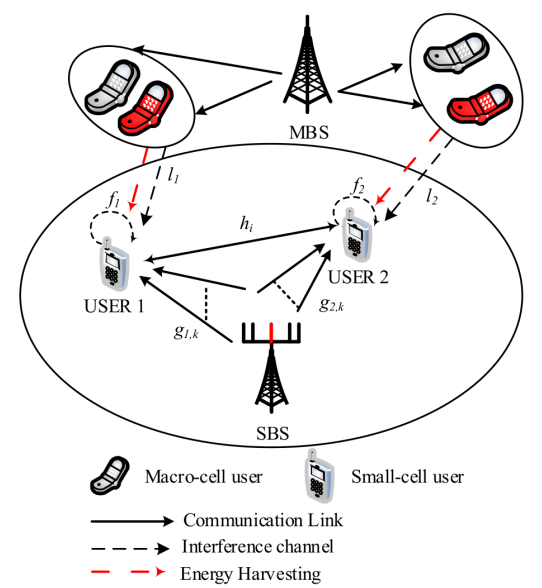

(c) $\mathrm{EH}$ from $\mathrm{MCU}$

Fig. 2: The three practical scenarios of small-cell NOMA network.

The outage probability at the non-SIC user 1 in this scheme 
given by [40]

$$
\begin{aligned}
& O P_{D 1}^{f-s 1}=\underbrace{\operatorname{Pr}\left(\gamma_{1, k^{*}}^{s 1}<\varepsilon_{1}^{f}, \gamma_{1 \leftarrow 2, k^{*}}^{s 1}<\varepsilon_{1}^{f}\right)}_{A_{1}} \\
& +\underbrace{\operatorname{Pr}\left(\max \left\{\gamma_{1, k^{*}}^{s 1}, \chi_{1}^{s 1}\right\}<\varepsilon_{1}^{f}, \gamma_{1 \leftarrow 2, k^{*}}^{s 1}>\varepsilon_{1}^{f}\right)}_{A_{2}} .
\end{aligned}
$$

Proposition 1: The outage probability at user 1 in closedform $O P_{D 1}^{f-s 1}$ is computed by

$$
\begin{aligned}
& O P_{D 1}^{f-s 1}=\left(1-\xi_{1}^{f}\right)\left(1-\xi_{2}^{f}\right)+\left(1-\xi_{1}^{f}\right) \\
& \times\left[1-\frac{\lambda_{h_{1}}}{\lambda_{h_{1}}+\varepsilon_{1}^{f} \varpi \lambda_{f_{1}}} \exp \left(-\frac{\varepsilon_{1}^{f}}{\rho \lambda_{h_{1}}}\right)\right] \xi_{2}^{f},
\end{aligned}
$$

where $\varepsilon_{i}^{f}=2^{R_{i}}-1, \varepsilon_{i}^{h}=2^{2 R_{i}}-1, \psi^{q}=\frac{\varepsilon_{1}^{q}}{\rho\left(a_{1}-\varepsilon_{1}^{q} a_{2}\right)}, \xi_{i}^{q}=$ $\sum_{k=1}^{K}\left(\begin{array}{l}K \\ k\end{array}\right)(-1)^{k-1} \exp \left(-\frac{\psi^{q} k}{\lambda_{i}}\right), q=f$ or $h$.

Proof: Please refer to Appendix A.

\section{B. Outage probability of user 1 in Scheme 1 using HD mode}

Based on results from (9) and (10) outage probability of user 1 in Scheme 1 can be computed as

$$
\begin{aligned}
& O P_{D 1}^{h-s 1}=\left(1-\xi_{1}^{h}\right)\left(1-\xi_{2}^{h}\right)+\left(1-\xi_{1}^{h}\right) \\
& \times\left[1-\exp \left(-\frac{\varepsilon_{1}^{h}}{\rho \lambda_{h_{1}}}\right)\right] \xi_{2}^{h} .
\end{aligned}
$$

\section{Outage probability of user 2 in Scheme 1 using FD mode}

Consider the outage probability of $D_{2}$ in small-cell network employing NOMA. If the outage event does not happen at $D_{1}$, then $D_{2}$ can more opportunity to receive cooperation signal transmitted from $D_{1}$. It is noted that $D_{2}$ requires SIC process. In worse case, transmission from $D_{1}$ to $D_{2}$ in bidirectional link cannot be performed if the $D_{1}$ 's data rate is less than $R_{D 1}^{s 1}$. As a result [40], outage probability of $D_{2}$ is expressed as

$$
\begin{aligned}
& O P_{D 2}^{f-s 1}=\underbrace{\operatorname{Pr}\left[\begin{array}{c}
\left(\gamma_{2, k^{*}}^{s 1}<\varepsilon_{2}^{F D} \cup \gamma_{1 \leftarrow 2, k^{*}}^{s 1}<\varepsilon_{1}^{f}\right) \\
\gamma_{1, k^{*}}^{s 1}<\varepsilon_{2}^{f}
\end{array}\right]}_{B_{1}} \\
& +\underbrace{\operatorname{Pr}\left[\begin{array}{c}
\gamma_{2, k^{*}}^{s 1}<\varepsilon_{2}^{f} \cup \max \left\{\gamma_{1 \leftarrow 2, k^{*}}^{s 1}, \chi_{2}^{s 1}\right\}<\varepsilon_{1}^{f}, \\
\gamma_{1, k^{*}}^{s 1}>\varepsilon_{1}^{f}
\end{array}\right]}_{B_{2}} .
\end{aligned}
$$

Proposition 2: The closed-form formula to show the outage probability $O P_{D 2}^{f-s 1}$ at $D_{2}$ is

$$
\begin{aligned}
& O P_{D 2}^{f-s 1}=\left[1-\sum_{k=1}^{K}\left(\begin{array}{c}
K \\
k
\end{array}\right)(-1)^{k-1} \exp \left(-\frac{\theta^{f} k}{\lambda_{2}}\right)\right] \\
& \times\left[1-\sum_{k=1}^{K}\left(\begin{array}{c}
K \\
k
\end{array}\right)(-1)^{k-1} \exp \left(-\frac{\delta^{f} k}{\lambda_{1}}\right)\right] \\
& +\left[\left(1-\mu_{1}^{f}\right)+\left(1-\xi_{2}^{f}\right)\left(1-\eta_{1}^{f}\right)\right. \\
& \left.-\left(1-\mu_{1}^{f}\right)\left(1-\xi_{2}^{f}\right)\left(1-\eta_{1}^{f}\right)\right] \xi_{1}^{f},
\end{aligned}
$$

where $\theta^{q}=\max \left(\frac{\varepsilon_{2}^{q}}{a_{2} \rho}, \psi^{q}\right), \quad \delta^{q}=\frac{\varepsilon_{2}^{q}}{\rho\left(a_{1}-\varepsilon_{2}^{q} a_{2}\right)}$, $\mu_{1}^{q}=\sum_{k=1}^{K}\left(\begin{array}{l}K \\ k\end{array}\right)(-1)^{k-1} \exp \left(-\frac{\varepsilon_{2}^{q} k}{a_{2} \rho \lambda_{2}}\right), \quad \eta_{1}^{f}=$ $\frac{\lambda_{h_{2}}}{\lambda_{h_{2}}+\varepsilon_{1}^{f} \varpi \lambda_{f_{2}}} \exp \left(-\frac{\varepsilon_{1}^{f}}{\rho \lambda_{h_{2}}}\right), \eta_{1}^{h}=\exp \left(-\frac{\varepsilon_{1}^{h}}{\rho \lambda_{h_{2}}}\right)$.

Proof: Please refer to Appendix B.

\section{Outage probability of user $D_{2}$ in Scheme 1 using $H D$ mode}

In similar way, it can be obtained outage probability $D_{2}$ in scheme 1 at HD mode as

$$
\begin{aligned}
& O P_{D 2}^{h-s 1}=\left[1-\sum_{k=1}^{K}\left(\begin{array}{l}
K \\
k
\end{array}\right)(-1)^{k-1} \exp \left(-\frac{\theta^{h} k}{\lambda_{2}}\right)\right] \\
& \times\left[1-\sum_{k=1}^{K}\left(\begin{array}{l}
K \\
k
\end{array}\right)(-1)^{k-1} \exp \left(-\frac{\delta^{h} k}{\lambda_{1}}\right)\right] \\
& +\left[\left(1-\mu_{1}^{h}\right)+\left(1-\xi_{2}^{h}\right)\left(1-\eta_{1}^{h}\right)\right. \\
& \left.-\left(1-\mu_{1}^{h}\right)\left(1-\xi_{2}^{h}\right)\left(1-\eta_{1}^{h}\right)\right] \xi_{1}^{h} .
\end{aligned}
$$

\section{SCHEME 2: IMPACT OF INTERFERENCE FROM MCU ON BIDIRECTIONAL USERS}

In case of existence of interference from the MCU to two NOMA users, the received signal can be obtained at $D_{i},(i=1,2)$

$$
y_{D i}^{s 2}=g_{i, k}\left(\sqrt{a_{1} P_{S}} x_{1}+\sqrt{a_{2} P_{s}} x_{2}\right)+\sqrt{P_{R}} l_{i} x_{R}+w_{i} .
$$

Then, the received SINRs at user $D_{1}$ is computed as

$$
\gamma_{1, k}^{s 2}=\frac{a_{1} \rho\left|g_{1, k}\right|^{2}}{a_{2} \rho\left|g_{1, k}\right|^{2}+\rho\left|l_{1}\right|^{2}+1} .
$$

The SINR for SIC user to detect $x_{1}$ and the received SINRs at user $D_{2}$ is given by

$$
\gamma_{1 \leftarrow 2, k}^{s 2}=\frac{a_{1} \rho\left|g_{2, k}\right|^{2}}{a_{2} \rho\left|g_{2, k}\right|^{2}+\rho\left|l_{2}\right|^{2}+1}, \gamma_{2, k}^{s 2}=\frac{a_{2} \rho\left|g_{2, k}\right|^{2}}{\rho\left|l_{2}\right|^{2}+1} .
$$

In this phase, the user with a stronger channel gain and the user with a weaker gain need cooperate. Designing bidirectional link can assist user $D_{1}$ to decode its data, or user $D_{2}$ to operate SIC better. In such situation, received signal obtained by user $D_{i}$ is expressed as

$$
z_{D i}^{s 2}=\sqrt{P_{D i}} h_{i} x_{i}+\sqrt{\varpi P_{D i}} f_{i} x_{F D I}+\sqrt{P_{R}} l_{i} x_{R}+w_{i} .
$$

Then, the received SINR at user $D_{i}$ is given by

$$
\chi_{i}^{s 2}=\frac{\rho\left|h_{i}\right|^{2}}{\varpi \rho\left|f_{i}\right|^{2}+\rho\left|l_{i}\right|^{2}+1} .
$$

A. Outage probability of user 1 in Scheme 2 using FD mode

In Scheme 2, the outage probability of user 1 in FD mode is given as

$$
\begin{aligned}
& O P_{D 1}^{f-s 2}=\underbrace{\operatorname{Pr}\left(\gamma_{1, k^{*}}^{s 2}<\varepsilon_{1}^{f}, \gamma_{1 \leftarrow 2, k^{*}}^{s 2}<\varepsilon_{1}^{f}\right)}_{C_{1}} \\
& +\underbrace{\operatorname{Pr}\left(\max \left\{\gamma_{1, k^{*}}^{s 2}, \chi_{1}^{s 2}\right\}<\varepsilon_{1}^{f}, \gamma_{1 \leftarrow 2, k^{*}}^{s 2}>\varepsilon_{1}^{f}\right)}_{C_{2}} .
\end{aligned}
$$


Proposition 3: The outage probability $O P_{D 1}^{f-s 2}$ at user $D_{1}$ can be formulated by

$$
\begin{aligned}
& O P_{D 1}^{f-s 2}=\left(1-\vartheta_{1}^{f}\right)\left(1-\vartheta_{2}^{f}\right)+\left(1-\vartheta_{1}^{f}\right) \vartheta_{2}^{f} \\
& \times\left[1-\frac{\lambda_{h_{1}}}{\varepsilon_{1}^{f} \varpi \lambda_{f_{1}}+\lambda_{h_{1}}} \frac{\lambda_{h_{1}}}{\varepsilon_{1}^{f} \lambda_{l_{1}}+\lambda_{h_{1}}} \exp \left(-\frac{\varepsilon_{1}^{f}}{\rho \lambda_{h_{1}}}\right)\right],
\end{aligned}
$$

where $\vartheta_{i}^{q}=\sum_{k=1}^{K}\left(\begin{array}{c}K \\ k\end{array}\right)(-1)^{k-1} \frac{\left(a_{1}-\varepsilon_{1}^{q} a_{2}\right) \lambda_{i}}{k \varepsilon_{1}^{q} \lambda_{l_{i}}+\left(a_{1}-\varepsilon_{1}^{q} a_{2}\right) \lambda_{i}} \exp \left(-\frac{\psi^{q} k}{\lambda_{i}}\right)$, $i \in\{1,2\}$.

Proof: Please refer to Appendix C.

\section{B. Outage probability of user 1 in Scheme 2 using HD mode}

Lack of self-interference channel due to FD mode, the considered system in HD mode results in other form of outage probability and then relying on results in (20) and (21), outage probability $D_{1}$ in Scheme 2 at HD mode is formulated as

$$
\begin{array}{r}
O P_{D 1}^{h-s 2}=\left(1-\vartheta_{1}^{h}\right)\left(1-\vartheta_{2}^{h}\right)+\left(1-\vartheta_{1}^{h}\right) \vartheta_{2}^{h} \\
\times\left[1-\frac{\lambda_{h_{1}}}{\varepsilon_{1}^{h} \lambda_{l_{1}}+\lambda_{h_{1}}} \exp \left(-\frac{\varepsilon_{1}^{h}}{\rho \lambda_{h_{1}}}\right)\right] .
\end{array}
$$

C. Outage probability of user 2 in Scheme 2 using FD mode

The outage probability of user 2 in Scheme 2 at FD mode can be computed and final result is presented in the following proposition.

$$
\left.\begin{array}{l}
O P_{D 2}^{f-s 2}=\operatorname{Pr}\left[\left(\gamma_{2, k^{*}}^{s 2}<\varepsilon_{2}^{f} \cup \gamma_{1 \leftarrow 2, k^{*}}^{s 2}<\varepsilon_{1}^{f}\right), \gamma_{1, k^{*}}^{s 2}<\varepsilon_{2}^{f}\right. \\
+\operatorname{Pr}\left(\gamma_{2, k^{*}}^{s 2}<\varepsilon_{2}^{f} \cup \max \left\{\gamma_{1 \leftarrow 2, k^{*}}^{s 2}, \chi_{2}^{s 2}\right\}<\varepsilon_{1}^{f}, \gamma_{1, k^{*}}^{s 2}>\varepsilon_{1}^{f}\right.
\end{array}\right\}
$$

Proposition 4: The closed-form expression of the outage probability $O P_{D 2}^{f-s 2}$ is formulated as

$$
\begin{aligned}
& O P_{D 2}^{f-s 2}=\left(1-\Omega_{1}^{f}\right)\left(1-\Omega_{2}^{f}\right) \\
& +\left[\left(1-\wp_{1}^{f}\right)+\left(1-\wp_{2}^{f}\right)\left(1-\vartheta_{2}^{f}\right)\right. \\
& \left.-\left(1-\wp_{1}^{f}\right)\left(1-\vartheta_{2}^{f}\right)\left(1-\wp_{2}^{f}\right)\right] \vartheta_{1}^{f},
\end{aligned}
$$

where $\Omega_{1}^{q}=\sum_{k=1}^{K}\left(\begin{array}{l}K \\ k\end{array}\right)(-1)^{k-1} \frac{\lambda_{2}}{\theta^{q} k \rho \lambda_{l_{2}}+\lambda_{2}} \exp \left(-\frac{\theta^{q} k}{\lambda_{2}}\right)$, $\Omega_{2}^{q}=\sum_{k=1}^{K}\left(\begin{array}{l}K \\ k\end{array}\right)(-1)^{k-1} \frac{\left(a_{1}-\varepsilon_{2}^{q} a_{2}\right) \lambda_{1}}{k \varepsilon_{2}^{q} \lambda_{1}+\left(a_{1}-\varepsilon_{2}^{q} a_{2}\right) \lambda_{1}} \exp \left(-\frac{\delta^{q} k}{\lambda_{1}}\right)$, $\wp_{1}^{q}=\sum_{k=1}^{K}\left(\begin{array}{l}K \\ k\end{array}\right)(-1)^{k-1} \frac{a_{2} \lambda_{2}}{a_{2} \lambda_{2}+\varepsilon_{2}^{q} k \lambda_{l_{2}}} \exp \left(-\frac{\varepsilon_{2}^{q} k}{a_{2} \rho \lambda_{2}}\right), \wp_{2}^{f}=$ $\frac{\lambda_{h_{2}}}{\varepsilon_{1}^{f} \varpi \lambda_{f_{2}}+\lambda_{h_{2}}} \frac{\lambda_{h_{2}}}{\varepsilon_{1}^{f} \lambda_{l_{2}}+\lambda_{h_{2}}} \exp \left(-\frac{\varepsilon_{1}^{f}}{\rho \lambda_{h_{2}}}\right)$.

Proof: Please refer to Appendix D.

\section{Outage probability of user 2 in Scheme 2 with HD mode}

Considering HD mode, such outage probability can be achieved straightforward. Using results in (23) and (24), outage probability $D_{2}$ in scheme 2 at HD mode is formulated as

$$
\begin{aligned}
& O P_{D 2}^{h-s 2}=\left(1-\Omega_{1}^{h}\right)\left(1-\Omega_{2}^{h}\right) \\
& +\left[\left(1-\wp_{1}^{h}\right)+\left(1-\wp_{2}^{h}\right)\left(1-\vartheta_{2}^{h}\right)\right. \\
& \left.-\left(1-\wp_{1}^{h}\right)\left(1-\vartheta_{2}^{h}\right)\left(1-\wp_{2}^{h}\right)\right] \vartheta_{1}^{h},
\end{aligned}
$$

in which $\wp_{2}^{h}=\frac{\lambda_{h_{2}}}{\varepsilon_{1}^{h} \lambda_{l_{2}}+\lambda_{h_{2}}} \exp \left(-\frac{\varepsilon_{1}^{h}}{\rho \lambda_{h_{2}}}\right)$.

\section{SCHEME 3: EH-ASSISTED USERS}

In this scheme, once MCU is wireless power enabler, $D_{i}$ can harvest energy from the MCU in the first phase and uses such energy to transmit the signal to $D_{i}$ in the second phase. It is assumed that the energy harvested from the noise can be ignored. Therefore, according to the power splitting protocol (PS) [41, 42] for $\mathrm{EH}$, the received signal at $D_{i}$ in the first phase is expressed as

$$
\begin{aligned}
y_{D i}^{s 3} & =g_{i, k}\left(\sqrt{a_{1} P_{S}} x_{1}+\sqrt{a_{2} P_{s}} x_{2}\right)+\sqrt{(1-\beta) P_{R}} l_{i} x_{R} \\
& +w_{i} .
\end{aligned}
$$

Then the received SINRs at user $D_{1}$ is computed as

$$
\gamma_{1, k}^{s 3}=\frac{a_{1} \rho\left|g_{1, k}\right|^{2}}{a_{2} \rho\left|g_{1, k}\right|^{2}+(1-\beta) \rho\left|l_{1}\right|^{2}+1} .
$$

The SINR for SIC user to detect $x_{1}$ and the received SINRs at user $D_{2}$ is given by

$$
\begin{aligned}
\gamma_{1 \leftarrow 2, k}^{s 3} & =\frac{a_{1} \rho\left|g_{2, k}\right|^{2}}{a_{2} \rho\left|g_{2, k}\right|^{2}+(1-\beta) \rho\left|l_{2}\right|^{2}+1}, \\
\gamma_{2, k}^{s 3} & =\frac{a_{2} \rho\left|g_{2, k}\right|^{2}}{(1-\beta) \rho\left|l_{2}\right|^{2}+1} .
\end{aligned}
$$

It is assumed that the energy harvested from the noise can be ignored $[42,43]$ and the harvest energy measured at bidirectional users are expressed by $E=\frac{T}{2} \eta \beta\left(P_{R}\left|l_{i}\right|^{2}+P_{D i}\left|f_{i}\right|^{2}\right)$. As a result, the transmit power $\frac{E}{T / 2}$ at user $D_{i}$ can be expressed as $P_{D i}=\frac{\eta \beta P_{R}\left|l_{i}\right|^{2}}{1-\eta \beta\left|f_{i}\right|^{2}}[42,43]$.

In this phase, $D_{i}$ uses such energy from MCU to transmit the signal, the user with a stronger channel gain and the user with a weaker gain need cooperate. In such situation, received signal obtained by user $D_{i}$ is expressed as [41, 42]

$$
\begin{aligned}
z_{D i}^{s 3} & =\sqrt{P_{D i}} h_{i} x_{i}+\sqrt{\varpi P_{D i}} f_{i} x_{F D I}+\sqrt{(1-\beta) P_{R}} l_{i} x_{R} \\
& +w_{i} .
\end{aligned}
$$

The received SINR at $D_{i}$ in this circumstance is expressed by

$$
\chi_{i}^{s 3}=\frac{\eta \beta \rho\left|l_{i}\right|^{2}\left|h_{i}\right|^{2}}{\varpi \eta \beta \rho\left|l_{i}\right|^{2}\left|f_{i}\right|^{2}+(1-\beta)\left(1-\eta \beta\left|f_{i}\right|^{2}\right) \rho\left|l_{i}\right|^{2}+\left(1-\eta \beta\left|f_{i}\right|^{2}\right)} .
$$

A. Outage probability of user 1 in Scheme 3 using FD mode

In this situation, the outage probability user 1 in Scheme 3 is defined by

$$
\begin{aligned}
& O P_{D 1}^{f-s 3}=\underbrace{\operatorname{Pr}\left(\gamma_{1, k^{*}}^{s 3}<\varepsilon_{1}^{f}, \gamma_{1 \leftarrow 2, k^{*}}^{s 3}<\varepsilon_{1}^{f}\right)}_{E_{1}} \\
& +\underbrace{\operatorname{Pr}\left(\max \left\{\gamma_{1, k^{*}}^{s 3}, \chi_{1}^{s 3}\right\}<\varepsilon_{1}^{f}, \gamma_{1 \leftarrow 2, k^{*}}^{s 3}>\varepsilon_{1}^{f}\right)}_{E_{2}} .
\end{aligned}
$$

Proposition 5: The exact expression is derived to indicate the outage probability $O P_{D 1}^{f-s 3}$ as

$O P_{D 1}^{f-s 3}=\left(1-\Phi_{1}^{f}\right)\left(1-\Phi_{2}^{f}\right)+\left(1-\Phi_{1}^{f}\right) \Phi_{2}^{f}\left(1-\Upsilon_{1}^{f}\right)$, 
where $\Phi_{i}^{q}=\sum_{k=1}^{K}\left(\begin{array}{l}K \\ k\end{array}\right)(-1)^{k-1} \frac{\left(a_{1}-\varepsilon_{1}^{q} a_{2}\right) \lambda_{i}}{(1-\beta) k \varepsilon_{1}^{q} \lambda_{l_{i}}+\left(a_{1}-\varepsilon_{1}^{q} a_{2}\right) \lambda_{i}}$ $\exp \left(-\frac{\psi^{q} k}{\lambda_{i}}\right), \Upsilon_{i}^{f}=\frac{1}{\lambda_{l_{i}}} \exp \left(-\frac{(1-\beta) \varepsilon_{1}^{f}}{\eta \beta \lambda_{h_{i}}}\right)$ $\int_{0}^{\infty} \frac{\rho \lambda_{h_{i}} y}{(\varpi-1+\beta) \varepsilon_{1}^{f} \rho \lambda_{f_{i}} y-\varepsilon_{1}^{f} \lambda_{f_{i}}+\rho \lambda_{h_{i}} y} \exp \left(-\frac{\varepsilon_{1}^{f}}{\eta \beta \rho \lambda_{h_{i}} y}-\frac{y}{\lambda_{l_{i}}}\right) d y$.

Proof: Please refer to Appendix E.

\section{B. Outage probability of user 1 in Scheme 3 using HD mode}

It is assumed that the energy harvested from the noise can be ignored [42, 43] and the harvest energy measured at bidirectional users for HD are expressed by $E_{h}=\frac{T}{2} \eta \beta P_{R}\left|l_{i}\right|^{2}$. Then, the transmitted power $\frac{E_{h}}{T / 2}$ at $D_{1}$ for HD can be expressed as $P_{D i}=\eta \beta P_{R}\left|l_{i}\right|^{2}$.

In this phase, $D_{i}$ uses such energy from MCU to transmit the signal, the user with a stronger channel gain and the user with a weaker gain need cooperate. In such situation, received signal obtained by user $D_{i}$ for HD is expressed as $z_{D i}^{h-s 3}=\sqrt{P_{D i}} h_{i} x_{i}+\sqrt{(1-\beta) P_{R}} l_{i} x_{R}+w_{i}$ [41, 42]. Then, the received SINR at $D_{i}$ in this circumstance is expressed by $\chi_{i}^{h-s 3}=\frac{\eta \beta \rho\left|l_{i}\right|^{2}\left|h_{i}\right|^{2}}{(1-\beta) \rho\left|l_{i}\right|^{2}+1}$.

In this situation, the outage probability user 1 in Scheme 3 for HD is defined by

$$
\begin{aligned}
& O P_{D 1}^{h-s 3}=\underbrace{\operatorname{Pr}\left(\gamma_{1, k^{*}}^{s 3}<\varepsilon_{1}^{h}, \gamma_{1 \leftarrow 2, k^{*}}^{s 3}<\varepsilon_{1}^{h}\right)}_{F_{1}} \\
& +\underbrace{\operatorname{Pr}\left(\max \left\{\gamma_{1, k^{*}}^{s 3}, \chi_{1}^{h-s 3}\right\}<\varepsilon_{1}^{h}, \gamma_{1 \leftarrow 2, k^{*}}^{s 3}>\varepsilon_{1}^{h}\right)}_{F_{2}} .
\end{aligned}
$$

From (33), $F_{1}$ is computed similarly as $E_{1}, F_{1}$ is given by $F_{1}=\left(1-\Phi_{1}^{h}\right)\left(1-\Phi_{2}^{h}\right)$. By using this way, $F_{2}$ can be calculated by

$$
F_{2}=\underbrace{\operatorname{Pr}\left(\gamma_{1, k^{*}}^{s 3}<\varepsilon_{1}^{h}\right)}_{F_{2 a}} \underbrace{\operatorname{Pr}\left(\chi_{1}^{h-s 3}<\varepsilon_{1}^{h}\right)}_{F_{2 b}} \underbrace{\operatorname{Pr}\left(\gamma_{1 \leftarrow 2, k^{*}}^{s 3}>\varepsilon_{1}^{h}\right)}_{F_{2 c}},
$$

where $F_{2 a}$ and $F_{2 c}$ in (34) can be expressed as $F_{1}$. Furthermore, $F_{2 b}$ can be calculated as

$$
\begin{aligned}
& F_{2 b}=1-\operatorname{Pr}\left(\left|h_{1}\right|^{2} \geq \frac{\varepsilon_{1}^{h}\left((1-\beta) \rho\left|l_{1}\right|^{2}+1\right)}{\eta \beta \rho\left|l_{1}\right|^{2}}\right) \\
& =1-\int_{0}^{\infty}\left(1-F_{\left|h_{1}\right|^{2}}\left(\frac{\varepsilon_{1}^{h}((1-\beta) \rho x+1)}{\eta \beta \rho x}\right)\right) f_{\left|l_{1}\right|^{2}}(x) d x \\
& =1-\frac{1}{\lambda_{l_{1}}} \exp \left(-\frac{\varepsilon_{1}^{h}(1-\beta)}{\eta \beta \lambda_{h_{1}}}\right) \int_{0}^{\infty} \exp \left(-\frac{\varepsilon_{1}^{h}}{\eta \beta \rho \lambda_{h_{1}} x}-\frac{x}{\lambda_{l_{1}}}\right) d x \\
& =1-\Upsilon_{1}^{h},
\end{aligned}
$$

where $\Upsilon_{i}^{h}=\exp \left(-\frac{\varepsilon_{1}^{h}(1-\beta)}{\eta \beta \lambda_{h_{i}}}\right) \sqrt{\frac{4 \varepsilon_{1}^{h}}{\eta \beta \rho \lambda_{h_{i}} \lambda_{l_{i}}}} K_{1}\left(\sqrt{\frac{4 \varepsilon_{1}^{h}}{\eta \beta \rho \lambda_{h_{i}} \lambda_{l_{i}}}}\right)$.

Substituting $F_{2 a}, F_{2 c}$ and (35) into (34), $F_{2}$ can be written as $F_{2}=\left(1-\Phi_{1}^{h}\right) \Phi_{2}^{h}\left(1-\Upsilon_{1}^{h}\right)$.

By combining $F_{1}$ and $F_{2}$ into (33), the outage probability performance of $D_{1}$ in Scheme 3 at HD mode can be formulated by

$$
O P_{D 1}^{h-s 3}=\left(1-\Phi_{1}^{h}\right)\left(1-\Phi_{2}^{h}\right)+\left(1-\Phi_{1}^{h}\right) \Phi_{2}^{h}\left(1-\Upsilon_{1}^{h}\right) .
$$

\section{Outage probability of user 2 in Scheme 3 using FD}

Proposition 6: The outage probability user $D_{2}$ in scheme 3 at FD mode can be addressed as

$$
\begin{aligned}
& O P_{D 2}^{f-s 3}=\left(1-\Theta_{1}^{f}\right)\left(1-\Theta_{2}^{f}\right) \\
& +\left[\left(1-\Xi_{1}^{f}\right)+\left(1-\Upsilon_{2}^{f}\right)\left(1-\Phi_{2}^{f}\right)\right. \\
& \left.-\left(1-\Xi_{1}^{f}\right)\left(1-\Phi_{2}^{f}\right)\left(1-\Upsilon_{2}^{f}\right)\right] \Phi_{1}^{f},
\end{aligned}
$$

in which $\Theta_{1}^{q}=\sum_{k=1}^{K}\left(\begin{array}{l}K \\ k\end{array}\right)(-1)^{k-1} \frac{\lambda_{2}}{(1-\beta) \theta^{q} k \rho \lambda_{l_{2}}+\lambda_{2}} \exp \left(-\frac{\theta^{q} k}{\lambda_{2}}\right)$, $\Theta_{2}^{q}=\sum_{k=1}^{K}\left(\begin{array}{l}K \\ k\end{array}\right)(-1)^{k-1} \frac{\left(a_{1}-\varepsilon_{2}^{q} a_{2}\right) \lambda_{1}}{(1-\beta) k \varepsilon_{2}^{q} \lambda_{l_{1}}+\left(a_{1}-\varepsilon_{2}^{q} a_{2}\right) \lambda_{1}} \exp \left(-\frac{\delta^{q} k}{\lambda_{1}}\right)$, $\Xi_{1}^{q}=\sum_{k=1}^{K}\left(\begin{array}{l}K \\ k\end{array}\right)(-1)^{k-1} \frac{a_{2} \lambda_{2}}{a_{2} \lambda_{2}+(1-\beta) \varepsilon_{2}^{q} k \lambda_{l_{2}}} \exp \left(-\frac{\varepsilon_{2}^{q} k}{a_{2} \rho \lambda_{2}}\right)$.

Proof: See in Appendix F.

\section{Outage probability user 2 in Scheme 3 using HD}

Based on (35), (37) it can be achieved outage probability $D_{2}$ in scheme 3 with HD mode as

$$
\begin{aligned}
& O P_{D 2}^{h-s 3}=\left(1-\Theta_{1}^{h}\right)\left(1-\Theta_{2}^{h}\right) \\
& +\left[\left(1-\Xi_{1}^{h}\right)+\left(1-\Upsilon_{2}^{h}\right)\left(1-\Phi_{2}^{h}\right)\right. \\
& \left.-\left(1-\Xi_{1}^{h}\right)\left(1-\Phi_{2}^{h}\right)\left(1-\Upsilon_{2}^{h}\right)\right] \Phi_{1}^{h} .
\end{aligned}
$$

\section{E. Asymptotic Analysis}

In order to obtain more insights, we analyze the asymptotic outage probability performance at high SNR by using $\rho \rightarrow \infty$. The asymptotic outage probability of $D_{1}$ and $D_{2}$ for FD/HD are given by

$$
\begin{aligned}
O P_{D 1}^{\infty-s 3}= & \left(1-\Phi_{1}^{\infty}\right)\left(1-\Phi_{2}^{\infty}\right)+\left(1-\Phi_{1}^{\infty}\right) \Phi_{2}^{\infty}\left(1-\Upsilon_{1}^{\infty}\right), \\
& O P_{D 2}^{\infty-s 3}=\left(1-\Theta_{1}^{\infty}\right)\left(1-\Theta_{2}^{\infty}\right) \\
& +\left[\left(1-\Xi_{1}^{\infty}\right)+\left(1-\Upsilon_{2}^{\infty}\right)\left(1-\Phi_{2}^{\infty}\right)\right. \\
& \left.-\left(1-\Xi_{1}^{\infty}\right)\left(1-\Phi_{2}^{\infty}\right)\left(1-\Upsilon_{2}^{\infty}\right)\right] \Phi_{1}^{\infty},
\end{aligned}
$$

where $\Phi_{1}^{\infty}=\sum_{k=1}^{K}\left(\begin{array}{l}K \\ k\end{array}\right)(-1)^{k-1} \frac{\left(a_{1}-\varepsilon_{1}^{q} a_{2}\right) \lambda_{1}}{(1-\beta) k \varepsilon_{1}^{q} \lambda_{l_{1}}+\left(a_{1}-\varepsilon_{1} a_{2}\right) \lambda_{1}}$, $\Phi_{2}^{\infty}=\sum_{k=1}^{K}\left(\begin{array}{l}K \\ k\end{array}\right)(-1)^{k-1} \frac{\left(a_{1}-\varepsilon_{1}^{q} a_{2}\right) \lambda_{2}}{(1-\beta) \varepsilon_{1}^{q} k \lambda_{l_{2}}+\left(a_{1}-\varepsilon_{1}^{q} a_{2}\right) \lambda_{2}}, \Upsilon_{1}^{\infty}=$ $\exp \left(-\frac{(1-\beta) \varepsilon_{1}^{q}}{\eta \beta \lambda_{h_{1}}}\right), \Theta_{1}^{\infty}=\sum_{k=1}^{K}\left(\begin{array}{l}K \\ k\end{array}\right)(-1)^{k-1} \frac{\lambda_{2}}{(1-\beta) \theta^{q} k \rho \lambda_{l_{2}}+\lambda_{2}}$, $\Theta_{2}^{\infty}=\sum_{k=1}^{K}\left(\begin{array}{l}K \\ k\end{array}\right)(-1)^{k-1} \frac{\left(a_{1}-\varepsilon_{2}^{q} a_{2}\right) \lambda_{1}}{(1-\beta) k \varepsilon_{2}^{q} \lambda_{l_{1}}+\left(a_{1}-\varepsilon_{2}^{q} a_{2}\right) \lambda_{1}}, \quad \Xi_{1}^{\infty}=$ $\sum_{k=1}^{K}\left(\begin{array}{l}K \\ k\end{array}\right)(-1)^{k-1} \frac{a_{2} \lambda_{2}}{a_{2} \lambda_{2}+(1-\beta) \varepsilon_{2}^{q} k \lambda_{l_{2}}}, \Upsilon_{2}^{\infty}=\exp \left(-\frac{(1-\beta) \varepsilon_{1}^{q}}{\eta \beta \lambda_{h_{2}}}\right)$.

\section{F. The overall outage probability of the system for 3 Schemes}

The overall outage probability of the system can be given by

$$
O P_{S Y S T E M}^{q-s}=1-\left(1-O P_{D 1}^{q-s}\right)\left(1-O P_{D 2}^{q-s}\right),
$$

where $s=s 1, s 2, s 3$. 
TABLE III: Main parameters for our simulations.

\begin{tabular}{|l|l|l|}
\hline Parameters & Notation & Values \\
\hline Power coefficients & $\left\{a_{1}, a_{2}\right\}$ & $\{0.8,0.2\}$ \\
\hline $\begin{array}{l}\text { Target rates SINR to decode } \\
x_{1} \text { and } x_{2}\end{array}$ & $R_{1}=R_{2}$ & 1 (BPCU) \\
\hline $\begin{array}{l}\text { Energy conversion coefficient } \\
{[41,42]}\end{array}$ & $\eta$ & 0.6 \\
\hline $\begin{array}{l}\text { The power splitting ratio [41, } \\
\text { 42] }\end{array}$ & $\beta$ & 0.6 \\
\hline Path loss exponent & $\alpha$ & 2 \\
\hline $\begin{array}{l}\text { Normalized distances from } \\
\text { SBS to } D_{1} \text { and SBS to } D_{2}\end{array}$ & $\left\{d_{1}, d_{2}\right\}$ & $\{0.4,0.2\}$ \\
\hline $\begin{array}{l}\text { Average powers } \\
\left\{\lambda_{1}=d_{1}^{\Theta *},\right. \\
\lambda_{2}=d_{2}^{\Theta *},\end{array}$ & $\left\{0.4^{2}, 0.2^{2}, 1\right\}$ \\
$\lambda_{l_{1}}=\lambda_{l_{2}}=$ & \\
$\lambda_{f_{1}}=\lambda_{f_{2},}$, & \\
\hline $\begin{array}{l}\text { Conversion operation betwen } \\
\text { FD/HD }\end{array}$ & $\varpi$ & 1 \\
\hline $\begin{array}{l}\text { Number of transmission anten- } \\
\text { nas }\end{array}$ & $K$ & 5 \\
\hline
\end{tabular}

\section{NumERiCAL Results AND Discussions}

In this section, We evaluate performance of communication between the SBS and two users $D_{1}, D_{2}$. The main parameters can be considered in Table III except for specific cases. We will verify results achieved from mathematical analysis, then they exhibit several necessary comparisons.

First, in Fig. 3(a), we present the outage probability of a two user in a group in small-cell network with respect to transmit SNR at SBS. It can be seen tight matching curves as comparing Monte Carlo simulation with analytic results. It can be concluded that performance gap among two users is resulted from different power level allocated to users. It is intuitively seen that higher $a_{1}$ provides better performance for user $D_{1}$. At each user, FD mode exhibits better outage performance compared with that in HD mode. As a result, it is important as selecting different operation mode for user in such small-cell network according to the different SNR levels in practical systems. In Fig. 3(b), it can be seen that the required rate at $D_{2}$ make impact on its performance. In this case, increasing transmit SNR provides outage improvement, especially at high SNR outage probability is very small. Other trends of curves can be observed in similar manner as in Fig. 3(a). Fig. 3(c) plots outage probability versus transmit SNR at the SBS $\rho$ at two cases related to the number of transmit antennas, $K=1,5$. It can be explained that improved outage performance achieved since more antennas are equipped at the SBS. Through this simulation, it can be seen how the number of antennas at the SBS affects the outage performance significantly. It can be confirmed that the best outage performance occurs at the case $K=5$ for user $D_{1}$ in FD mode.

Fig. 4(a) plots the outage probability of FD/HD NOMA with interference link from MCUs as varying $a_{1}$ from 0.5 to 1 . It is concluded that optimal outage performance of user $D_{2}$ can be achieved at several values of $a_{1}$ for FD and HD cases. Fig. 4(b) indicates that outage performance decrease significantly at high requirement of $R_{1}$. The reason is that outage behavior is limited by target rates. These curves for outage performance

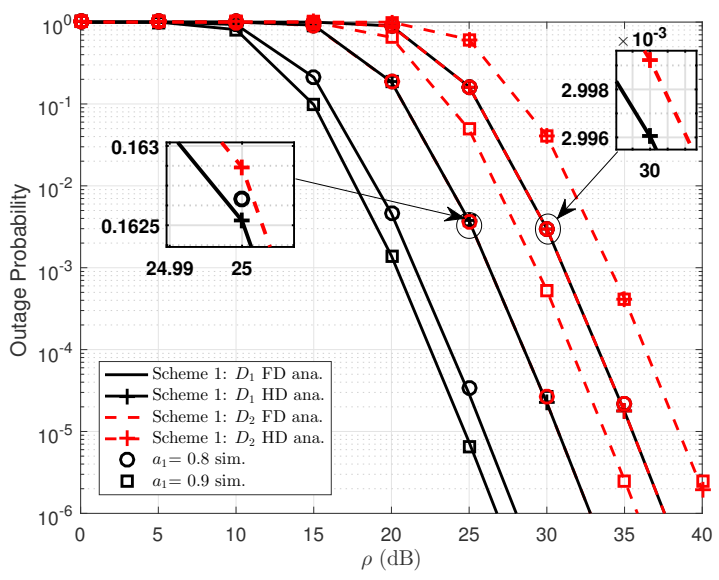

(a) Varying power factor $a_{1}$

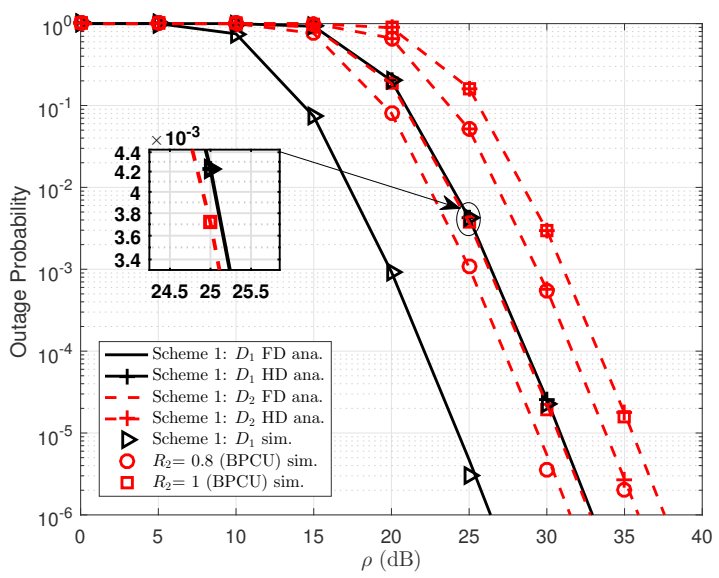

(b) Varying target rates $R_{2}$

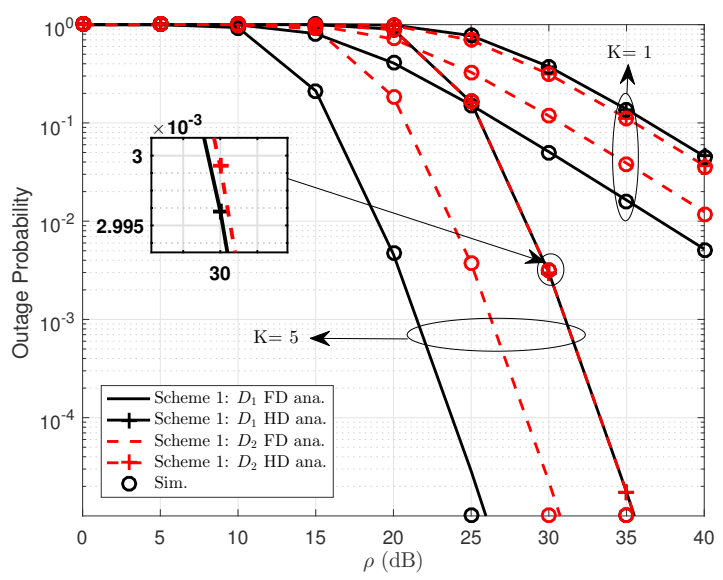

(c) Varying $K$

Fig. 3: Scheme 1: Outage probability comparison between FD and HD for user $D_{1}, D_{2}$ versus $\rho$.

for HD case become saturation as $R_{1}$ is greater than 1.15 . In contrast, outage performance will worse at higher value of target rate $R_{1}$. This situation can be further explained that degraded performance is resulted by interference originated from MCUs in Scheme 2. 


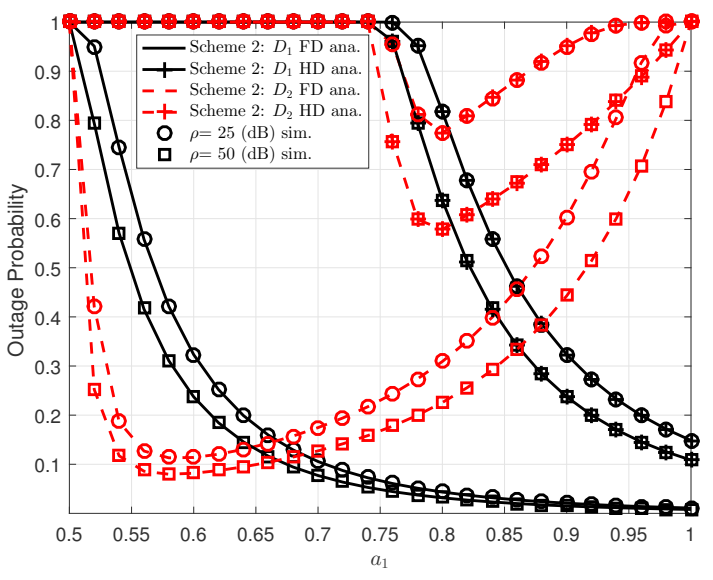

(a) Versus $a_{1}$ as varying $\rho$

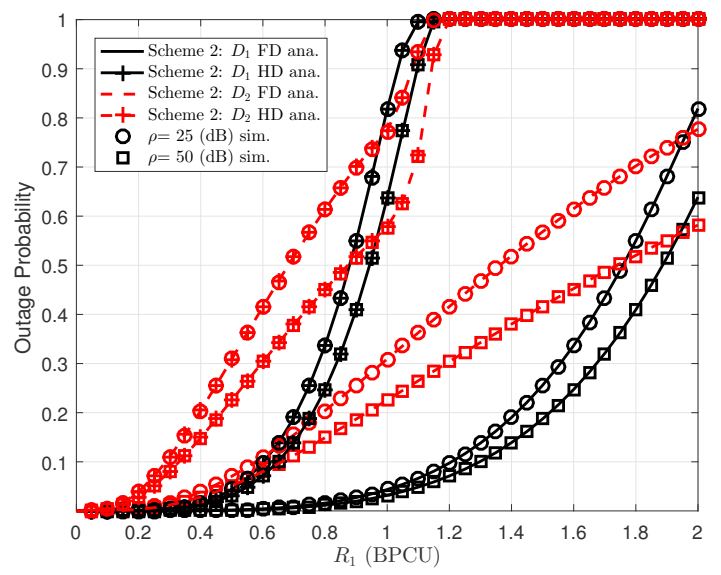

(b) Versus $R_{1}=R_{2}$ as varying $\rho$

Fig. 4: Scheme 2: Outage probability comparison between FD and HD for user $D_{1}, D_{2}$.

Fig. 5(a) and Fig. 5(b) demonstrate outage performance of two users in Scheme 3 for FD and HD cases. The better outage performance is recognized for user $D_{1}$ at the point $\lambda_{h_{1}}=$ $\lambda_{h_{2}}=0.01$. It is also confirmed strong channels exhibit better outage performance, as shown in Fig. 5(b). Similarly, Fig. 5(c) shows how amount of harvested power leads to performance improvement achieved for both users.

Fig. 6(a) depicts that the outage performance of Scheme 1 and Scheme 2 exhibit large gap among two schemes. This situation is related impact of interference from macro-cell network. It is confirmed that challenge happens as important problem for HetNet in term of inter-cell interference. Therefore, limiting the impact of interference in Scheme 2 help to remain outage performance at acceptable level for small-cell network. Fig. 6(b) also reports that the outage performance with $R_{1}=R_{2}=0.1(\mathrm{bps} / \mathrm{Hz})$ in Scheme 3 worse than that in Scheme 1 significantly. While Fig. 6(b) confirms that outage probability of user $D_{1}$ in Scheme 1 outperforms than that in Scheme 3. In contrast, user $D_{2}$ in Scheme 3 is better than that in Scheme 2 in term of outage probability. In addition, small-cell NOMA exhibits its advances compared with the counterpart (OMA scheme). Finally, Fig. 6(c) depicts the

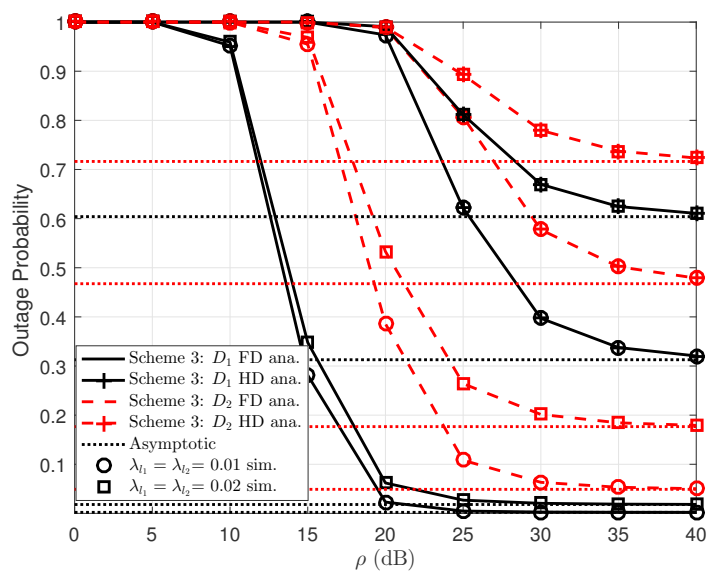

(a) Varying $\lambda_{l_{1}}=\lambda_{l_{2}}$

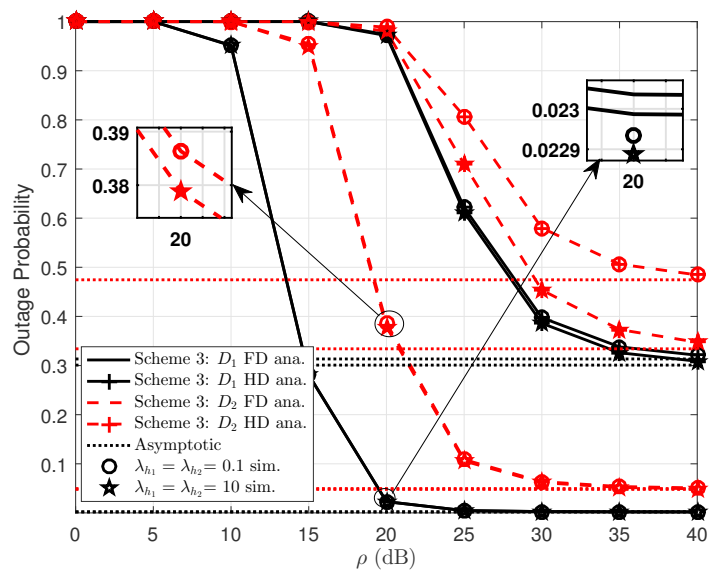

(b) Varying channel power $\lambda_{h_{1}}=\lambda_{h_{2}}$

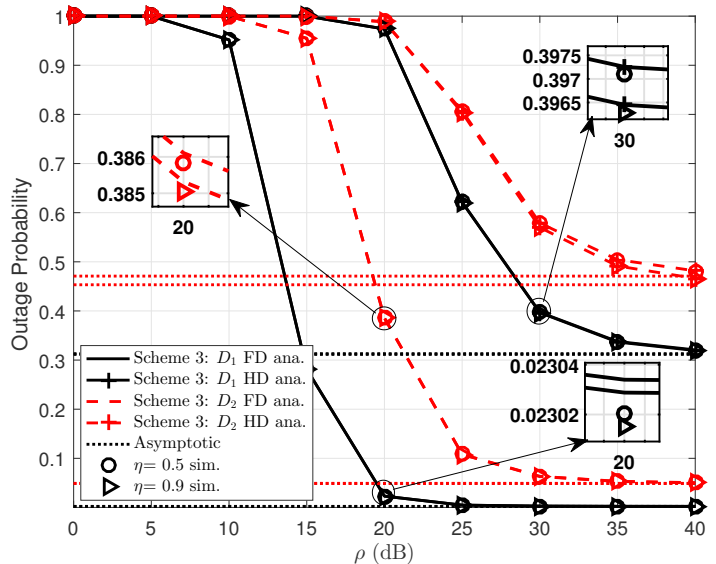

(c) Varying $\eta$

Fig. 5: Scheme 3: Outage probability comparison between FD and HD for user $D_{1}, D_{2}$ versus $\rho$.

most improvement of outage performance as observed for user $D_{1}$ Scheme 3. It can be explained that EH along higher power coefficient certainly are crucial impacts on performance improvement for cell-edge user. 


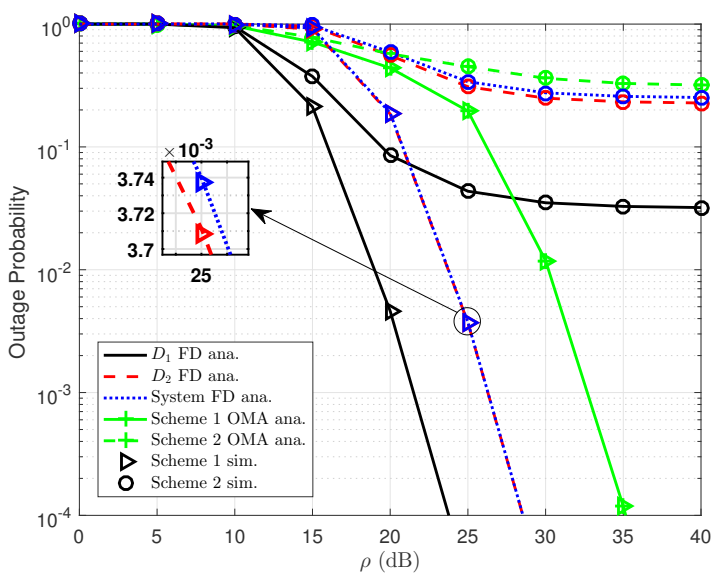

(a) Between Scheme 1, Scheme 2 and OMA

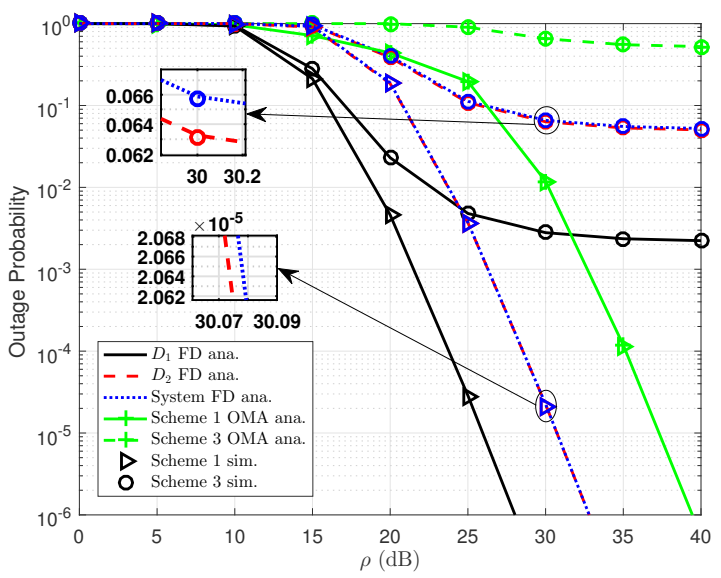

(b) Between Scheme 1, Scheme 3 and OMA

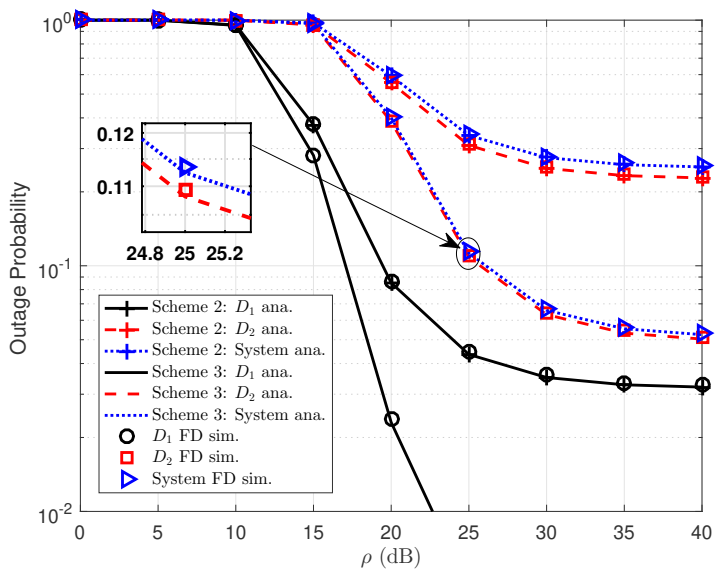

(c) Between Scheme 2 and Scheme 3

Fig. 6: Comparison of outage performance.

\section{CONCLUSION}

In this paper, the downlink NOMA system is studied to allow two users communicate each other directly. In this paper, multiple antennas are well equipped at the BS to provide higher diversity for destinations which have better opportunity to select suitable signal. To prolong lifetime of such destinations, linear EH protocol is enabled. It is confirmed that system performance related to three proposed schemes are still retained. In particular, Scheme 1 exhibits its superiority at high SNR compared with remaining schemes. As most important result. The closed-form expressions of outage probability were derived for the two destinations to evaluate the system performance of the proposed scheme, and several comparisons regarding power allocation factor, number of transmit antenna at the BS were provided as well. Simulation results were presented to indicate that the analytical results of outage behavior under impact of interference from MCUs (Scheme 2 and Scheme 3) will become worse compared with ideal scenario in Scheme 1. More importantly, the proposed HetNet NOMA schemes can significantly improve the outage performance by enabling more antennas at the BS and capable of $\mathrm{EH}$.

\section{APPENDIX A}

\section{PROOF OF PROPOSITION 1}

From (9), $A_{1}$ can be computed by

$$
\begin{aligned}
& A_{1}=\operatorname{Pr}\left(\gamma_{1, k^{*}}^{s 1}<\varepsilon_{1}^{f}\right) \operatorname{Pr}\left(\gamma_{1 \leftarrow 2, k^{*}}^{s 1}<\varepsilon_{1}^{f}\right) \\
& =\operatorname{Pr}\left(\left|g_{1, k^{*}}\right|^{2}<\frac{\varepsilon_{1}^{f}}{\rho\left(a_{1}-\varepsilon_{1}^{f} a_{2}\right)}\right) \operatorname{Pr}\left(\left|g_{2, k^{*}}\right|^{2}<\frac{\varepsilon_{1}^{f}}{\rho\left(a_{1}-\varepsilon_{1}^{f} a_{2}\right)}\right) \\
& =\operatorname{Pr}\left(\left|g_{1, k^{*}}\right|^{2}<\psi^{f}\right) \operatorname{Pr}\left(\left|g_{2, k^{*}}\right|^{2}<\psi^{f}\right) \\
& =F_{\left|g_{1, k^{*}}\right|^{2}}\left(\psi^{f}\right) F_{\left|g_{2, k^{*}}\right|^{2}}\left(\psi^{f}\right),
\end{aligned}
$$

where $\psi^{f}=\frac{\varepsilon_{1}^{f}}{\rho\left(a_{1}-\varepsilon_{1}^{f} a_{2}\right)}$. Based on (7), $A_{1}$ is given by $A_{1}=\left(1-\xi_{1}^{f}\right)\left(1-\xi_{2}^{f}\right)$, where $\xi_{i}^{q}=\sum_{k=1}^{K}\left(\begin{array}{l}K \\ k\end{array}\right)(-1)^{k-1} \exp \left(-\frac{\psi^{q} k}{\lambda_{i}}\right)$.

From (9) and based on $A_{1}, A_{2}$ can be expressed as

$$
\begin{aligned}
& A_{2}=\operatorname{Pr}\left(\gamma_{1, k^{*}}^{s 1}<\varepsilon_{1}^{f}\right) \operatorname{Pr}\left(\chi_{1}^{s 1}<\varepsilon_{1}^{f}\right) \operatorname{Pr}\left(\gamma_{1 \leftarrow 2, k^{*}}^{s 1}>\varepsilon_{1}^{f}\right) \\
& =\left(1-\xi_{1}^{f}\right) \underbrace{\operatorname{Pr}\left(\chi_{1}^{s 1}<\varepsilon_{1}^{f}\right)}_{A_{2 a}} \xi_{2}^{f} .
\end{aligned}
$$

Furthermore, $A_{2 a}$ can be calculated as

$$
\begin{aligned}
& A_{2 a}=1-\operatorname{Pr}\left(\left|h_{1}\right|^{2} \geq \frac{\varepsilon_{1}^{f} \varpi \rho\left|f_{1}\right|^{2}+\varepsilon_{1}^{f}}{\rho}\right) \\
& =1-\int_{0}^{\infty}\left(1-F_{\left|h_{1}\right|^{2}}\left(\frac{\varepsilon_{1}^{f} \varpi \rho x+\varepsilon_{1}^{f}}{\rho}\right)\right) f_{\left|f_{1}\right|^{2}}(x) d x .
\end{aligned}
$$

The CDF and PDF of channel $\left|g_{i, k}\right|^{2}$ are based on (7) and (8), respectively. It is noted that the other channels, i.e., $\left|h_{i}\right|^{2}$, $\left|l_{i}\right|^{2}$ and all loop feedback channels follow the Rayleigh distribution with CDF and PDF are $F_{|X|^{2}}(x)=1-\exp \left(-\frac{x}{\lambda_{X}}\right)$, $f_{|X|^{2}}(x)=\frac{1}{\lambda_{X}} \exp \left(-\frac{x}{\lambda_{X}}\right)$, respectively. It is straightforward to obtain

$$
\begin{aligned}
& A_{2 a}=1-\int_{0}^{\infty} \exp \left(-\frac{\varepsilon_{1}^{f} \varpi \rho x+\varepsilon_{1}^{f}}{\rho \lambda_{h_{1}}}\right) \frac{1}{\lambda_{f_{1}}} \exp \left(-\frac{x}{\lambda_{f_{1}}}\right) d x \\
& =1-\frac{\lambda_{h_{1}}}{\lambda_{h_{1}}+\varepsilon_{1}^{f} \varpi \lambda_{f_{1}}} \exp \left(-\frac{\varepsilon_{1}^{f}}{\rho \lambda_{h_{1}}}\right) .
\end{aligned}
$$

Substituting (A.4) into (A.2), $A_{2}$ is written as $A_{2}=$ $\left(1-\xi_{1}^{f}\right)\left[1-\frac{\lambda_{h_{1}}}{\lambda_{h_{1}}+\varepsilon_{1}^{f} \varpi \lambda_{f_{1}}} \exp \left(-\frac{\varepsilon_{1}^{f}}{\rho \lambda_{h_{1}}}\right)\right] \xi_{2}^{f}$. 
Combining $A_{1}$ and $A_{2}$ into (9), we can obtain (10). It completes the proof.

\section{APPENDIX B}

\section{PROOF OF PROPOSITION 2}

From (12), $B_{1}$ is computed by

$B_{1}=[1-\underbrace{\operatorname{Pr}\left(\gamma_{2, k^{*}}^{s 1} \geq \varepsilon_{2}^{f}, \gamma_{1 \leftarrow 2, k^{*}}^{s 1} \geq \varepsilon_{1}^{f}\right)}_{B_{1 a}}] \underbrace{\operatorname{Pr}\left(\gamma_{1, k^{*}}^{s 1}<\varepsilon_{2}^{f}\right)}_{B_{1 b}}$.

(B.1)

By using (B.1), $B_{1 a}$ can be evaluated as

$$
\begin{aligned}
& B_{1 a}=\operatorname{Pr}\left(\left|g_{2, k^{*}}\right|^{2} \geq \frac{\varepsilon_{2}^{f}}{a_{2} \rho},\left|g_{2, k^{*}}\right|^{2} \geq \psi^{f}\right) \\
& =\operatorname{Pr}\left(\left|g_{2, k^{*}}\right|^{2} \geq \max \left(\frac{\varepsilon_{2}^{f}}{a_{2} \rho}, \psi^{f}\right)\right) \\
& =\operatorname{Pr}\left(\left|g_{2, k^{*}}\right|^{2} \geq \theta^{f}\right)=1-F_{\left|g_{2, k^{*}}\right|^{2}}\left(\theta^{f}\right) \\
& =\sum_{k=1}^{K}\left(\begin{array}{l}
K \\
k
\end{array}\right)(-1)^{k-1} \exp \left(-\frac{\theta^{f} k}{\lambda_{2}}\right),
\end{aligned}
$$

where $\theta^{f}=\max \left(\frac{\varepsilon_{2}^{f}}{a_{2} \rho}, \psi^{f}\right)$ and note that (B.2) is obtained by using the $\mathrm{CDF}$ in (7).

Similarly, $B_{1 b}$ can be rewritten as

$$
\begin{aligned}
& B_{1 b}=\operatorname{Pr}\left(\left|g_{1, k^{*}}\right|^{2}<\frac{\varepsilon_{2}^{f}}{\rho\left(a_{1}-\varepsilon_{2}^{f} a_{2}\right)}\right)=F_{\left|g_{1, k^{*}}\right|^{2}}\left(\delta^{f}\right) \\
& =1-\sum_{k=1}^{K}\left(\begin{array}{l}
K \\
k
\end{array}\right)(-1)^{k-1} \exp \left(-\frac{\delta^{f} k}{\lambda_{1}}\right),
\end{aligned}
$$

where $\delta^{f}=\frac{\varepsilon_{2}^{f}}{\rho\left(a_{1}-\varepsilon_{2}^{f} a_{2}\right)}$.

Substituting (B.2) and (B.3) into (B.1), $B_{1}$ is written as

$$
\begin{aligned}
& B_{1}=\left(1-\sum_{k=1}^{K}\left(\begin{array}{l}
K \\
k
\end{array}\right)(-1)^{k-1} \exp \left(-\frac{\theta^{f} k}{\lambda_{2}}\right)\right) \\
& \times\left(1-\sum_{k=1}^{K}\left(\begin{array}{l}
K \\
k
\end{array}\right)(-1)^{k-1} \exp \left(-\frac{\delta^{f} k}{\lambda_{1}}\right)\right) .
\end{aligned}
$$

Based on (12) and $A_{1}$, we can rewrite $B_{2}$ as

$$
\begin{aligned}
& B_{2}=[\underbrace{\operatorname{Pr}\left(\gamma_{2, k^{*}}^{s 1}<\varepsilon_{2}^{f}\right)}_{B_{21}}+\underbrace{\operatorname{Pr}\left(\max \left\{\gamma_{1 \leftarrow 2, k^{*}}^{s 1}, \chi_{2}^{s 1}\right\}<\varepsilon_{1}^{f}\right)}_{B_{22}} \\
& -\underbrace{\operatorname{Pr}\left(\gamma_{2, k^{*}}^{s 1}<\varepsilon_{2}^{f} \cap \max \left\{\gamma_{1 \leftarrow 2, k^{*}}^{s 1}, \chi_{2}^{s 1}\right\}<\varepsilon_{1}^{f}\right)}_{B_{23}}] \xi_{1}^{f} .
\end{aligned}
$$

From (B.5), $B_{21}$ is given as

$$
\begin{aligned}
& B_{21}=\operatorname{Pr}\left(\gamma_{2, k^{*}}^{s 1}<\varepsilon_{2}^{f}\right)=1-\operatorname{Pr}\left(\left|g_{2, k^{*}}\right|^{2} \geq \frac{\varepsilon_{2}^{f}}{a_{2} \rho}\right) \\
& =1-\sum_{k=1}^{K}\left(\begin{array}{l}
K \\
k
\end{array}\right)(-1)^{k-1} \exp \left(-\frac{\varepsilon_{2}^{f} k}{a_{2} \rho \lambda_{2}}\right)=1-\mu_{1}^{f},
\end{aligned}
$$

where $\mu_{1}^{f}=\sum_{k=1}^{K}\left(\begin{array}{l}K \\ k\end{array}\right)(-1)^{k-1} \exp \left(-\frac{\varepsilon_{2}^{f} k}{a_{2} \rho \lambda_{2}}\right)$.
From (B.5) and based on (A.1), we can achieve $B_{22}$ as

$$
\begin{aligned}
& B_{22}=\operatorname{Pr}\left(\gamma_{1 \leftarrow 2, k^{*}}^{s 1}<\varepsilon_{1}^{f}, \chi_{2}^{s 1}<\varepsilon_{1}^{f}\right) \\
& =\operatorname{Pr}\left(\gamma_{1 \leftarrow 2, k^{*}}^{s 1}<\varepsilon_{1}^{f}\right) \operatorname{Pr}\left(\chi_{2}^{s 1}<\varepsilon_{1}^{f}\right) \\
& =\left(1-\xi_{2}^{f}\right) \underbrace{\operatorname{Pr}\left(\chi_{2}^{s 1}<\varepsilon_{1}^{f}\right)}_{B_{22 a}} .
\end{aligned}
$$

Then, $B_{22 a}$ can be simplified as

$$
\begin{aligned}
& B_{22 a}=1-\operatorname{Pr}\left(\chi_{2}^{s 1} \geq \varepsilon_{1}^{f}\right) \\
& =1-\operatorname{Pr}\left(\left|h_{2}\right|^{2} \geq \frac{\varepsilon_{1}^{f} \varpi \rho_{D}\left|f_{2}\right|^{2}+\varepsilon_{1}^{f}}{\rho_{D}}\right) \\
& =1-\int_{0}^{\infty}\left(1-F_{\left|h_{2}\right|^{2}}\left(\frac{\varepsilon_{1}^{f} \varpi \rho y+\varepsilon_{1}^{f}}{\rho}\right)\right) f_{\left|f_{2}\right|^{2}}(y) d y \\
& =1-\int_{0}^{\infty} \exp \left(-\frac{\varepsilon_{1}^{f} \varpi \rho y+\varepsilon_{1}^{f}}{\rho \lambda_{h_{2}}}\right) \frac{1}{\lambda_{f_{2}}} \exp \left(-\frac{y}{\lambda_{f_{2}}}\right) d y=1-\eta_{1}^{f},
\end{aligned}
$$

where $\eta_{1}^{f}=\frac{\lambda_{h_{2}}}{\lambda_{h_{2}}+\varepsilon_{1}^{F D} \varpi \lambda_{f_{2}}} \exp \left(-\frac{\varepsilon_{1}^{f}}{\rho \lambda_{h_{2}}}\right)$.

Substituting (B.8) into (B.7), $B_{22}$ is written as $B_{22}=$ $\left(1-\xi_{2}^{f}\right)\left(1-\eta_{1}^{f}\right)$.

Based on $B_{21}$ and $B_{22}, B_{23}$ can be expressed as

$$
\begin{aligned}
& B_{23}=\operatorname{Pr}\left(\gamma_{2, k^{*}}^{s 1}<\varepsilon_{2}^{f}\right) \operatorname{Pr}\left(\gamma_{1 \leftarrow 2, k^{*}}^{s 1}<\varepsilon_{1}^{f}\right) \operatorname{Pr}\left(\chi_{2}^{s 1}<\varepsilon_{1}^{f}\right) \\
& =\left(1-\mu_{1}^{f}\right)\left(1-\xi_{2}^{f}\right)\left(1-\eta_{1}^{f}\right) .
\end{aligned}
$$

By replacing $B_{21}, B_{22}$ and $B_{23}$ into (B.5). It can be further obtained $B_{2}$ as

$$
\begin{aligned}
& B_{2}=\left[\left(1-\mu_{1}^{f}\right)+\left(1-\xi_{2}^{f}\right)\left(1-\eta_{1}^{f}\right)\right. \\
& \left.-\left(1-\mu_{1}^{f}\right)\left(1-\xi_{2}^{f}\right)\left(1-\eta_{1}^{f}\right)\right] \xi_{1}^{f} .
\end{aligned}
$$

Combining (B.4), (B.10) into (12), we can obtain (13). It completes the proof.

\section{APPENDIX C}

\section{PROOF OF PROPOSITION 3}

From (20), $C_{1}$ is further computed as $C_{1}=$ $\underbrace{\operatorname{Pr}\left(\gamma_{1, k^{*}}^{s 2}<\varepsilon_{1}^{f}\right)}_{C_{1 a}} \underbrace{\operatorname{Pr}\left(\gamma_{1 \leftarrow 2, k^{*}}^{s 2}<\varepsilon_{1}^{f}\right)}_{C_{1 b}}$.

Then, $C_{1 a}$ can be obtained as

$$
\begin{aligned}
& C_{1 a}=1-\operatorname{Pr}\left(\gamma_{1, k^{*}}^{s 2} \geq \varepsilon_{1}^{f}\right) \\
& =1-\operatorname{Pr}\left(\left|g_{1, k^{*}}\right|^{2} \geq \frac{\varepsilon_{1}^{f} \rho\left|l_{1}\right|^{2}+\varepsilon_{1}^{f}}{\left(a_{1}-\varepsilon_{1}^{F D} a_{2}\right) \rho}\right) \\
& =1-\int_{0}^{\infty}\left(1-F_{\left|g_{1, k^{*}}\right|^{2}}\left(\frac{\varepsilon_{1}^{f} \rho x+\varepsilon_{1}^{f}}{\left(a_{1}-\varepsilon_{1}^{f} a_{2}\right) \rho}\right)\right) f_{\left|l_{1}\right|^{2}}(x) d x \\
& =1-\sum_{k=1}^{K}\left(\begin{array}{l}
K \\
k
\end{array}\right)(-1)^{k-1} \int_{0}^{\infty} \exp \left(-\frac{\left(\varepsilon_{1}^{f} \rho x+\varepsilon_{1}^{f}\right) k}{\left(a_{1}-\varepsilon_{1}^{f} a_{2}\right) \rho \lambda_{1}}\right) \\
& \times \frac{1}{\lambda_{l_{1}}} \exp \left(-\frac{x}{\lambda_{l_{1}}}\right) d x=1-\vartheta_{1}^{f},
\end{aligned}
$$


where $\vartheta_{i}^{q}=\sum_{k=1}^{K}\left(\begin{array}{c}K \\ k\end{array}\right)(-1)^{k-1} \frac{\left(a_{1}-\varepsilon_{1}^{q} a_{2}\right) \lambda_{i}}{k \varepsilon_{1}^{q} \lambda_{l_{i}}+\left(a_{1}-\varepsilon_{1}^{q} a_{2}\right) \lambda_{i}} \exp \left(-\frac{\psi^{q} k}{\lambda_{i}}\right)$. Similarly, $C_{1 b}^{k=1}$ is given by

$$
\begin{aligned}
& C_{1 b}=1-\operatorname{Pr}\left(\gamma_{1 \leftarrow 2, k^{*}}^{s 2} \geq \varepsilon_{1}^{f}\right) \\
& =1-\operatorname{Pr}\left(\left|g_{2, k^{*}}\right|^{2} \geq \frac{\varepsilon_{1} \rho\left|l_{2}\right|^{2}+\varepsilon_{1}^{f}}{\left(a_{1}-\varepsilon_{1}^{f} a_{2}\right) \rho}\right) \\
& =1-\int_{0}^{\infty}\left(1-F_{\left|g_{2, k^{*}}\right|^{2}}\left(\frac{\varepsilon_{1}^{f} \rho y+\varepsilon_{1}^{f}}{\left(a_{1}-\varepsilon_{1}^{f} a_{2}\right) \rho}\right)\right) f_{\left|l_{2}\right|^{2}}(y) d y \\
& =1-\sum_{k=1}^{K}\left(\begin{array}{l}
K \\
k
\end{array}\right)(-1)^{k-1} \int_{0}^{\infty} \exp \left(-\frac{\left(\varepsilon_{1} \rho y+\varepsilon_{1}^{f}\right) k}{\left(a_{1}-\varepsilon_{1}^{f} a_{2}\right) \rho \lambda_{2}}\right) \\
& \times \frac{1}{\lambda_{l_{2}}} \exp \left(-\frac{y}{\lambda_{l_{2}}}\right) d y=1-\vartheta_{2}^{f} .
\end{aligned}
$$

Base on (C.1) and (C.2), $C_{1}$ is written as $C_{1}=$ $\left(1-\vartheta_{1}^{f}\right)\left(1-\vartheta_{2}^{f}\right)$.

From (20), $C_{2}$ is further expressed as

$$
C_{2}=\underbrace{\operatorname{Pr}\left(\gamma_{1, k^{*}}^{s 2}<\varepsilon_{1}^{f}\right)}_{C_{2 a}} \underbrace{\operatorname{Pr}\left(\chi_{1}^{s 2}<\varepsilon_{1}^{f}\right)}_{C_{2 b}} \underbrace{\operatorname{Pr}\left(\gamma_{1 \leftarrow 2, k^{*}}^{s 2}>\varepsilon_{1}^{f}\right)}_{C_{2 c}} .
$$

Furthermore, $C_{2 a}$ and $C_{2 c}$ in (C.3) can be expressed as (C.1) and (C.2), respectively.

According to (C.3), $C_{2 b}$ can be calculated as follows

$$
\begin{aligned}
& C_{2 b}=1-\operatorname{Pr}\left(\chi_{1}^{s 2} \geq \varepsilon_{1}^{f}\right) \\
& =1-\operatorname{Pr}\left(\left|h_{1}\right|^{2} \geq \frac{\varepsilon_{1}^{f}\left(\varpi \rho\left|f_{1}\right|^{2}+\rho\left|l_{1}\right|^{2}+1\right)}{\rho}\right) \\
& =1-\int_{0}^{\infty} \int_{0}^{\infty}\left(1-F_{\left|h_{1}\right|^{2}}\left(\frac{\varepsilon_{1}^{f}(\varpi \rho x+\rho y+1)}{\rho}\right)\right) \\
& \times f_{\left|f_{1}\right|^{2}}(x) f_{\left|l_{1}\right|^{2}}(y) d x d y \\
& =1-\frac{1}{\lambda_{f_{1}}} \frac{1}{\lambda_{l_{1}}} \exp \left(-\frac{\varepsilon_{1}^{f}}{\rho \lambda_{h_{1}}}\right) \int_{0}^{\infty} \exp \left(-\left(\frac{\varepsilon_{1}^{f} \varpi}{\lambda_{h_{1}}}+\frac{1}{\lambda_{f_{1}}}\right) x\right) d x \\
& \times \int_{0}^{\infty} \exp \left(-\left(\frac{\varepsilon_{1}^{f}}{\lambda_{h_{1}}}+\frac{1}{\lambda_{l_{1}}}\right) y\right) d y \\
& =1-\frac{\lambda_{h_{1}}}{\varepsilon_{1}^{f} \varpi \lambda_{f_{1}}+\lambda_{h_{1}}} \frac{\lambda_{1}}{\varepsilon_{1}^{f} \lambda_{l_{1}}+\lambda_{h_{1}}} \exp \left(-\frac{\varepsilon_{1}^{f}}{\rho \lambda_{h_{1}}}\right) .
\end{aligned}
$$

By replacing (C.1), (C.2), (C.4) into (C.3) it can be obtained $C_{2}$ as

$$
C_{2}=\left(1-\vartheta_{1}^{f}\right) \vartheta_{2}^{f}\left[1-\frac{\lambda_{h_{1}}}{\varepsilon_{1}^{f} \varpi \lambda_{f_{1}}+\lambda_{h_{1}}} \frac{\lambda_{h_{1}}}{\varepsilon_{1}^{f} \lambda_{l_{1}}+\lambda_{h_{1}}} \exp \left(-\frac{\varepsilon_{1}^{f}}{\rho \lambda_{h_{1}}}\right)\right]
$$

Combining $C_{1}$, (C.5) into (20), we can obtain (21). It completes the proof.

APPENDIX D

\section{PROOF OF PROPOSITION 4}

From (23), $O P_{D 2}^{f-s 2}$ can given as

$$
\begin{aligned}
& O P_{D 2}^{f-s 2}=[1-\underbrace{\operatorname{Pr}\left(\gamma_{2, k^{*}}^{s 2} \geq \varepsilon_{2}^{f}, \gamma_{1 \leftarrow 2, k^{*}}^{s 2} \geq \varepsilon_{1}^{f}\right)}_{Q_{1}}] \\
& \times \underbrace{\operatorname{Pr}\left(\gamma_{1, k^{*}}^{s 2}<\varepsilon_{2}^{f}\right)}_{Q_{2}}+[\underbrace{\operatorname{Pr}\left(\gamma_{2, k^{*}}^{s 2}<\varepsilon_{2}^{f}\right)}_{Q_{3}} \\
& +\underbrace{\operatorname{Pr}\left(\max \left\{\gamma_{1 \leftarrow 2, k^{*}}^{s 2}, \chi_{2}^{s 2}\right\}<\varepsilon_{1}^{f}\right)}_{Q_{4}}
\end{aligned}
$$

$$
\begin{aligned}
& -\underbrace{\operatorname{Pr}\left(\gamma_{2, k^{*}}^{s 2}<\varepsilon_{2}^{f} \cap \max \left\{\gamma_{1 \leftarrow 2, k^{*}}^{s 2}, \chi_{2}^{s 2}\right\}<\varepsilon_{1}^{f}\right)}_{Q_{5}}] \\
& \times \underbrace{\operatorname{Pr}\left(\gamma_{1, k^{*}}^{s 2}>\varepsilon_{1}^{f}\right)}_{Q_{6}} .
\end{aligned}
$$

It is noted that $Q_{1}$ is further computed as

$$
\begin{aligned}
& Q_{1}=\operatorname{Pr}\left(\gamma_{S D 2, k^{*}}^{s 2} \geq \varepsilon_{2}^{f}, \gamma_{1 \leftarrow 2, k^{*}}^{s 2} \geq \varepsilon_{1}^{f}\right) \\
& =\operatorname{Pr}\left(\left|g_{2, k^{*}}\right|^{2} \geq \frac{\varepsilon_{2}^{f}\left(\rho\left|l_{2}\right|^{2}+1\right)}{a_{2} \rho},\left|g_{2, k^{*}}\right|^{2} \geq \frac{\varepsilon_{1}^{f}\left(\rho\left|l_{2}\right|^{2}+1\right)}{\rho\left(a_{1}-\varepsilon_{1} a_{2}\right)}\right) \\
& =\operatorname{Pr}\left(\left|g_{2, k^{*}}\right|^{2} \geq\left(\rho\left|l_{2}\right|^{2}+1\right) \theta^{f}\right) \\
& =\int_{0}^{\infty}\left(1-F_{\left|g_{2, k^{*}}\right|^{2}}\left((\rho y+1) \theta^{f}\right)\right) f_{\left|l_{2}\right|^{2}}(y) d y \\
& =\sum_{k=1}^{K}\left(\begin{array}{l}
K \\
k
\end{array}\right)(-1)^{k-1} \int_{0}^{\infty} \exp \left(-\frac{(\rho y+1) \theta^{f} k}{\lambda_{2}}\right) \frac{1}{\lambda_{l_{2}}} \exp \left(-\frac{y}{\lambda_{l_{2}}}\right) d y \\
& =\Omega_{1}^{f},
\end{aligned}
$$

where $\Omega_{1}^{f}=\sum_{k=1}^{K}\left(\begin{array}{l}K \\ k\end{array}\right)(-1)^{k-1} \frac{\lambda_{2}}{\theta^{f} k \rho \lambda_{l_{2}}+\lambda_{2}} \exp \left(-\frac{\theta^{f} k}{\lambda_{2}}\right)$. Similarly as $C_{1 a}, Q_{2}$ can be formulated as $Q_{2}=1-\Omega_{2}^{f}$, where $\Omega_{2}^{f}=\sum_{k=1}^{K}\left(\begin{array}{l}K \\ k\end{array}\right)(-1)^{k-1} \frac{\left(a_{1}-\varepsilon_{2}^{f} a_{2}\right) \lambda_{1}}{k \varepsilon_{2}^{f} \lambda_{l_{1}}+\left(a_{1}-\varepsilon_{2}^{f} a_{2}\right) \lambda_{1}} \exp \left(-\frac{\delta^{f} k}{\lambda_{1}}\right)$.

From (D.1), $Q_{3}$ is given by

$$
\begin{aligned}
& Q_{3}=1-\operatorname{Pr}\left(\left|g_{2, k^{*}}\right|^{2} \geq \frac{\varepsilon_{2}^{f} \rho\left|l_{2}\right|^{2}+\varepsilon_{2}^{f}}{a_{2} \rho}\right) \\
& =1-\int_{0}^{\infty}\left(1-F_{\left|g_{2, k^{*}}\right|^{2}}\left(\frac{\varepsilon_{2}^{f} \rho y+\varepsilon_{2}^{f}}{a_{2} \rho}\right)\right) f_{\left|l_{2}\right|^{2}}(y) d y \\
& =1-\sum_{k=1}^{K}\left(\begin{array}{l}
K \\
k
\end{array}\right)(-1)^{k-1} \int_{0}^{\infty} \exp \left(-\frac{\left(\varepsilon_{2}^{f} \rho y+\varepsilon_{2}^{f}\right) k}{a_{2} \rho \lambda_{2}}\right) \\
& \times \frac{1}{\lambda_{l_{2}}} \exp \left(-\frac{y}{\lambda_{l_{2}}}\right) d y=1-\wp_{1}^{f},
\end{aligned}
$$

where $\wp_{1}^{f}=\sum_{k=1}^{K}\left(\begin{array}{l}K \\ k\end{array}\right)(-1)^{k-1} \frac{a_{2} \lambda_{2}}{a_{2} \lambda_{2}+\varepsilon_{2}^{f} k \lambda_{l_{2}}} \exp \left(-\frac{\varepsilon_{2}^{f} k}{a_{2} \rho \lambda_{2}}\right)$.

Similarly as $C_{1 b}$ and $C_{2 b}$, It can be achieved $Q_{4}$ as

$$
\begin{aligned}
& Q_{4}=\left[1-\operatorname{Pr}\left(\chi_{2}^{s 2} \geq \varepsilon_{1}^{f}\right)\right]\left[1-\operatorname{Pr}\left(\gamma_{1 \leftarrow 2, k^{*}}^{s 2} \geq \varepsilon_{1}^{f}\right)\right] \\
& =\left(1-\wp_{2}^{f}\right)\left(1-\vartheta_{2}^{f}\right),
\end{aligned}
$$

where $\wp_{2}^{f}=\frac{\lambda_{h_{2}}}{\varepsilon_{1}^{f} \varpi \lambda_{f_{2}}+\lambda_{h_{2}}} \frac{\lambda_{h_{2}}}{\varepsilon_{1}^{f} \lambda_{l_{2}}+\lambda_{h_{2}}} \exp \left(-\frac{\varepsilon_{1}^{f}}{\rho \lambda_{h_{2}}}\right)$. 
By relying on $Q_{3}$ and $Q_{4}, Q_{5}$ can be given by

$$
\begin{aligned}
& Q_{5}=\operatorname{Pr}\left(\gamma_{2, k^{*}}^{s 2}<\varepsilon_{2}^{f} \cap \max \left\{\gamma_{1 \leftarrow 2, k^{*}}^{s 2}, \chi_{2}^{s 2}\right\}<\varepsilon_{1}^{f}\right) \\
& =\left[1-\operatorname{Pr}\left(\gamma_{2, k^{*}}^{s 2} \geq \varepsilon_{2}^{f}\right)\right]\left[1-\operatorname{Pr}\left(\gamma_{1 \leftarrow 2, k^{*}}^{s 2} \geq \varepsilon_{1}^{f}\right)\right] \\
& \times\left[1-\operatorname{Pr}\left(\chi_{2}^{s 2} \geq \varepsilon_{1}^{f}\right)\right] \\
& =\left(1-\wp_{1}^{f}\right)\left(1-\vartheta_{2}^{f}\right)\left(1-\wp_{2}^{f}\right) .
\end{aligned}
$$

Furthermore, $Q_{6}$ in (D.1) can be expressed as (C.1).

Combining (D.2)-(D.5) and (C.1) into (D.1), we can obtain (24). It completes the proof.

\section{APPENDIX E}

\section{PROOF OF PROPOSITION 5}

First, $\quad E_{1}$ is computed similarly as in $C_{1} . E_{1}$ is given by $E_{1}=\left(1-\Phi_{1}^{f}\right)\left(1-\Phi_{2}^{f}\right)$, where $\Phi_{i}^{q}=\sum_{k=1}^{K}\left(\begin{array}{l}K \\ k\end{array}\right)(-1)^{k-1} \frac{\left(a_{1}-\varepsilon_{1}^{q} a_{2}\right) \lambda_{i}}{(1-\beta) k \varepsilon_{1}^{q} \lambda_{l_{i}}+\left(a_{1}-\varepsilon_{1}^{q} a_{2}\right) \lambda_{i}} \exp \left(-\frac{\psi^{q} k}{\lambda_{i}}\right)$. While, ${ }^{k} \bar{E}_{2}$ is given by

$$
E_{2}=\underbrace{\operatorname{Pr}\left(\gamma_{1, k^{*}}^{s 3}<\varepsilon_{1}^{f}\right)}_{E_{2 a}} \underbrace{\operatorname{Pr}\left(\chi_{1}^{s 3}<\varepsilon_{1}^{f}\right)}_{E_{2 b}} \underbrace{\operatorname{Pr}\left(\gamma_{1 \leftarrow 2, k^{*}}^{s 3}>\varepsilon_{1}^{f}\right)}_{E_{2 c}},
$$

where $E_{2 a}$ and $E_{2 c}$ in (E.1) can be expressed as $E_{1}$. Furthermore, $E_{2 b}$ can be calculated as

$$
\begin{aligned}
& E_{2 b}=1-\operatorname{Pr}\left(\chi_{1}^{s 3} \geq \varepsilon_{1}^{f}\right) \\
& =1-\operatorname{Pr}\left(\left|h_{1}\right|^{2} \geq\right. \\
& \left.\frac{\varepsilon_{1}^{f}\left(\varpi \eta \beta \rho\left|l_{1}\right|^{2}\left|f_{1}\right|^{2}+(1-\beta)\left(1-\eta \beta\left|f_{1}\right|^{2}\right) \rho\left|l_{1}\right|^{2}+\left(1-\eta \beta\left|f_{1}\right|^{2}\right)\right)}{\eta \beta \rho\left|l_{1}\right|^{2}}\right) \\
& =1-\int_{0}^{\infty} \int_{0}^{\infty}(1- \\
& \left.F_{\left|h_{1}\right|^{2}}\left(-\frac{\varepsilon_{1}^{f} \varpi \eta \beta \rho x y+(1-\beta)(1-\eta \beta x) \varepsilon_{1}^{f} \rho y+\varepsilon_{1}^{f}(1-\eta \beta x)}{\eta \beta \rho y}\right)\right) \\
& \times f_{\left|f_{1}\right|^{2}}(x) f_{\left|l_{1}\right|^{2}}(y) d x d y \\
& =1-\int_{0}^{\infty} \int_{0}^{\infty} \exp \left(-\frac{\varepsilon_{1}^{f} \varpi \eta \beta \rho x y+(1-\beta)(1-\eta \beta x) \varepsilon_{1}^{f} \rho y+\varepsilon_{1}^{f}(1-\eta \beta x)}{\eta \beta \rho y \lambda_{h_{1}}}\right) \\
& \frac{1}{\lambda_{f_{1}}} \exp \left(-\frac{x}{\lambda_{f_{1}}}\right) \frac{1}{\lambda_{l_{1}}} \exp \left(-\frac{y}{\lambda_{l_{1}}}\right) d x d y=1-\Upsilon_{1}^{f},
\end{aligned}
$$

where $\Upsilon_{1}^{f}=\frac{1}{\lambda_{l_{1}}} \exp \left(-\frac{(1-\beta) \varepsilon_{1}^{f}}{\eta \beta \lambda_{h_{1}}}\right)$

$\int_{0}^{\infty} \frac{\rho \lambda_{h_{1}} y}{(\varpi-1+\beta) \varepsilon_{1}^{f} \rho \lambda_{f_{1}} y-\varepsilon_{1}^{f} \lambda_{f_{1}}+\rho \lambda_{h_{1}} y} \exp \left(-\frac{\varepsilon_{1}^{f}}{\eta \beta \rho \lambda_{h_{1}} y}-\frac{y}{\lambda_{l_{1}}}\right) d y$.

Substituting $E_{2 a}, E_{2 c}$ and (E.2) into (E.1), $E_{2}$ can written as $E_{2}=\left(1-\Phi_{1}^{f}\right) \Phi_{2}^{f}\left(1-\Upsilon_{1}^{f}\right)$.

Combining $E_{1}$ and $E_{2}$ into (31), we can obtain (32). It completes the proof.

\section{APPENDIX F}

\section{PROOF OF PROPOSITION 6}

Similarly as (23), The outage probability user $D_{2}$ in scheme 3 at FD mode can be expressed as

$$
\begin{aligned}
& O P_{D 2}^{f-s 3}=[1-\underbrace{\operatorname{Pr}\left(\gamma_{2, k^{*}}^{s 3} \geq \varepsilon_{2}^{f}, \gamma_{1 \leftarrow 2, k^{*}}^{s 3} \geq \varepsilon_{1}^{f}\right)}_{F_{1}}] \\
& \times \underbrace{\operatorname{Pr}\left(\gamma_{1, k^{*}}^{s 3}<\varepsilon_{2}^{f}\right)}_{F_{2}}+[\underbrace{\operatorname{Pr}\left(\gamma_{2, k^{*}}^{s 3}<\varepsilon_{2}^{f}\right)}_{F_{3}} \\
& +\underbrace{\operatorname{Pr}\left(\max \left\{\gamma_{1 \leftarrow 2, k^{*}}^{s 3}, \chi_{2}^{s 3}\right\}<\varepsilon_{1}^{f}\right)}_{F_{4}}) \\
& -\underbrace{\operatorname{Pr}\left(\gamma_{2, k^{*}}^{s 3}<\varepsilon_{2}^{f} \cap \max \left\{\gamma_{1 \leftarrow 2, k^{*}}^{s 3}, \chi_{2}^{s 3}\right\}<\varepsilon_{1}^{f}\right)}_{F_{5}}] \\
& \times \underbrace{\operatorname{Pr}\left(\gamma_{1, k^{*}}^{s 3}>\varepsilon_{1}^{f}\right)}_{F_{6}} .
\end{aligned}
$$

By exploiting $Q_{1}, F_{1}$ can be written as

$$
\begin{aligned}
& F_{1}=\operatorname{Pr}\left(\begin{array}{c}
\left|g_{2, k^{*}}\right|^{2} \geq \frac{\varepsilon_{2}^{f}\left((1-\beta) \rho\left|l_{2}\right|^{2}+1\right)}{a_{2} \rho}, \\
\left|g_{2, k^{*}}\right|^{2} \geq \frac{\varepsilon_{1}^{f}\left((1-\beta) \rho\left|l_{2}\right|^{2}+1\right)}{\rho\left(a_{1}-\varepsilon_{1} a_{2}\right)}
\end{array}\right) \\
& =\operatorname{Pr}\left(\left|g_{2, k^{*}}\right|^{2} \geq\left((1-\beta) \rho\left|l_{2}\right|^{2}+1\right) \theta^{f}\right) \\
& =\int_{0}^{\infty}\left(1-F_{\left|g_{2, k^{*}}\right|^{2}}\left(((1-\beta) \rho y+1) \theta^{f}\right)\right) f_{\left|l_{2}\right|^{2}}(y) d y \\
& =\sum_{k=1}^{K}\left(\begin{array}{l}
K \\
k
\end{array}\right)(-1)^{k-1} \int_{0}^{\infty} \exp \left(-\frac{((1-\beta) \rho y+1) \theta^{f} k}{\lambda_{2}}\right) \\
& \times \frac{1}{\lambda_{l_{2}}} \exp \left(-\frac{y}{\lambda_{l_{2}}}\right) d y=\Theta_{1}^{f},
\end{aligned}
$$

where $\Theta_{1}^{f}=\sum_{k=1}^{K}\left(\begin{array}{l}K \\ k\end{array}\right)(-1)^{k-1} \frac{\lambda_{2}}{(1-\beta) \theta^{f} k \rho \lambda_{l_{2}}+\lambda_{2}} \exp \left(-\frac{\theta^{f} k}{\lambda_{2}}\right)$.

Similarly as in $C_{1 a}$ and $Q_{2}, \quad F_{2}$ can given as $F_{2}=1-\Theta_{2}^{f}$, where $\Theta_{2}^{f}=$ $\sum_{k=1}^{K}\left(\begin{array}{l}K \\ k\end{array}\right)(-1)^{k-1} \frac{\left(a_{1}-\varepsilon_{2}^{f} a_{2}\right) \lambda_{1}}{(1-\beta) k \varepsilon_{2}^{f} \lambda_{l_{1}}+\left(a_{1}-\varepsilon_{2}^{f} a_{2}\right) \lambda_{1}} \exp \left(-\frac{\delta^{f} k}{\lambda_{1}}\right)$.

Furthermore, by using $Q_{3}, \quad F_{3}$ can be expressed by $F_{3}=1-\Xi_{1}^{f}$, where $\Xi_{1}^{f}=$ $\sum_{k=1}^{K}\left(\begin{array}{c}K \\ k\end{array}\right)(-1)^{k-1} \frac{a_{2} \lambda_{2}}{a_{2} \lambda_{2}+(1-\beta) \varepsilon_{2}^{f} k \lambda_{l_{2}}} \exp \left(-\frac{\varepsilon_{2}^{f} k}{a_{2} \rho \lambda_{2}}\right)$.

Furthermore, similarly as in $E_{2 b}$ and $E_{1} . F_{4}$ can written by

$$
\begin{aligned}
& F_{4}=\left[1-\operatorname{Pr}\left(\chi_{2}^{s 3} \geq \varepsilon_{1}^{f}\right)\right]\left[1-\operatorname{Pr}\left(\gamma_{1 \leftarrow 2, k^{*}}^{s 3} \geq \varepsilon_{1}^{f}\right)\right] \\
& =\left(1-\Upsilon_{2}^{f}\right)\left(1-\Phi_{2}^{f}\right),
\end{aligned}
$$

where $\Upsilon_{2}^{f}=\frac{1}{\lambda_{l_{2}}} \exp \left(-\frac{(1-\beta) \varepsilon_{1}^{f}}{\eta \beta \lambda_{h_{2}}}\right)$

$\int_{0}^{\infty} \frac{\rho \lambda_{h_{2}} y}{(\varpi-1+\beta) \varepsilon_{1}^{f} \rho \lambda_{f_{2}} y-\varepsilon_{1}^{f} \lambda_{f_{2}}+\rho \lambda_{h_{2}} y} \exp \left(-\frac{\varepsilon_{1}^{f}}{\eta \beta \rho \lambda_{h_{2}} y}-\frac{y}{\lambda_{l_{2}}}\right) d y$. Using $F_{3}$ and $F_{4}$, we obtain $F_{5}$ as

$$
\begin{aligned}
& F_{5}=\operatorname{Pr}\left(\gamma_{2, k^{*}}^{s 3}<\varepsilon_{2}^{f} \cap \max \left\{\gamma_{1 \leftarrow 2, k^{*}}^{s 3}, \chi_{2}^{s 3}\right\}<\varepsilon_{1}^{f}\right) \\
& =\left[1-\operatorname{Pr}\left(\gamma_{2, k^{*}}^{s 3} \geq \varepsilon_{2}^{f}\right)\right]\left[1-\operatorname{Pr}\left(\gamma_{1 \leftarrow 2, k^{*}}^{s 3} \geq \varepsilon_{1}^{f}\right)\right] \\
& \times\left[1-\operatorname{Pr}\left(\chi_{2}^{s 3} \geq \varepsilon_{1}^{f}\right)\right] \\
& =\left(1-\Xi_{1}^{f}\right)\left(1-\Phi_{2}^{f}\right)\left(1-\Upsilon_{2}^{f}\right) .
\end{aligned}
$$


Based on $E_{1}, F_{6}$ can formulated as $F_{6}=\Phi_{1}^{f}$. From (F.2)(F.4), $F_{2}, F_{3}$ and $F_{6}$ into (F.1), we can obtain (37), the proof is completed.

\section{REFERENCES}

[1] Y. Sun, Z. Ding, X. Dai, O. A. Dobre, "On the performance of network NOMA in uplink CoMP systems: A stochastic geometry approach," IEEE Transactions on Communications, vol. 67, no. 7, pp. 5084-5098, July 2019.

[2] Dinh-Thuan Do, M.-S. Van Nguyen, "Device-to-device transmission modes in NOMA network with and without Wireless Power Transfer," Computer Communications, vol. 139, pp. 67-77, May 2019.

[3] S. M. R. Islam, N. Avazov, O. A. Dobre, K. S. Kwak, "Power-Domain Non-Orthogonal Multiple Access (NOMA) in 5G Systems: Potentials and Challenges," IEEE Communications Surveys \& Tutorials, vol. 19, no. 2, pp. 721-742, Secondquarter 2017.

[4] Dinh-Thuan Do, A. Le, B. M. Lee, "NOMA in Cooperative Underlay Cognitive Radio Networks Under Imperfect SIC," IEEE Access, vol. 8, pp. 86180-86195, 2020.

[5] D.-T. Do, et al., "Throughput Analysis of Multipair TwoWay Replaying Networks With NOMA and Imperfect CSI," IEEE Access, vol. 8, pp. 128942-128953, 2020.

[6] Z. Yang, Z. Ding, P. Fan, N. Al-Dhahir, "A General Power Allocation Scheme to Guarantee Quality of Service in Downlink and Uplink NOMA Systems," IEEE Transactions on Wireless Communications, vol. 15, no. 11, pp. 7244-7257, Nov. 2016.

[7] J. Zhao, Y. Liu, K. K. Chai, Y. Chen, M. Elkashlan, "Joint subchannel and power allocation for NOMA enhanced D2D communications," IEEE Transactions on Communications, vol. 65, no. 11, pp. 5081-5094, Nov. 2017.

[8] N. Madani, S. Sodagari, "Performance Analysis of NonOrthogonal Multiple Access With Underlaid Device-toDeviceCommunications," IEEE Access, vol. 6, pp. 39820 - 39826, 2018.

[9] Y. Liu, Z. Qin, M. Elkashlan, A. Nallanathan, J. A. McCann, "Non-Orthogonal Multiple Access in LargeScale Heterogeneous Networks," IEEE Journal on Selected Areas in Communications, vol. 35, no. 12, pp. 2667-2680, Dec. 2017.

[10] C. Liu, D. Liang, P. Chen, J. Yang, "Coverage Analysis for Dense Heterogeneous Networks with Cooperative NOMA," 2017 IEEE 85th Vehicular Technology Conference (VTC Spring), 2017, pp. 1-6.

[11] Y. Wu, L. P. Qian, "Energy-Efficient NOMA-Enabled Traffic Offloading via Dual-Connectivity in Small-Cell Networks," IEEE Communications Letters, vol. 21, no. 7, pp. 1605-1608, July 2017.

[12] H. Wang, Y. Fu, Z. Shi, R. Song, "Fractional Power Control for Small Cell Uplinks with Opportunistic NOMA Transmissions," ICC 2019 - 2019 IEEE International Conference on Communications (ICC), Shanghai, China, 2019, pp. 1-7.
[13] F. Fang, J. Cheng, Z. Ding, "Joint Energy Efficient Subchannel and Power Optimization for a Downlink NOMA Heterogeneous Network," IEEE Transactions on Vehicular Technology, vol. 68, no. 2, pp. 1351-1364, Feb. 2019.

[14] L. P. Qian, Y. Wu, H. Zhou, X. Shen, "Joint Uplink Base Station Association and Power Control for Small-Cell Networks With Non-Orthogonal Multiple Access," IEEE Transactions on Wireless Communications, vol. 16, no. 9, pp. 5567-5582, Sept. 2017.

[15] Z. Ding, F. Adachi, H. V. Poor, "The application of MIMO to non-orthogonal multiple access", IEEE Transactions on Wireless Communications, vol. 15, no. 1, Jan. 2016.

[16] J. Ding, J. Cai, 'Two-Side Coalitional Matching Approach for Joint MIMO-NOMA Clustering and BS Selection in Multi-Cell MIMO-NOMA Systems," IEEE Transactions on Wireless Communications, vol. 19, no. 3, pp. 2006-2021, March 2020.

[17] Y. Liu, M. Zhao, L. Xiao, S. Zhou, "Pilot domain NOMA for grant-free massive random access in massive MIMO marine communication system," China Communications, vol. 17, no. 6, pp. 131-144, June 2020.

[18] A. A. Amin, S. Y. Shin, "Channel Capacity Analysis of Non-Orthogonal Multiple Access With OAM-MIMO System," IEEE Wireless Communications Letters, vol. 9, no. 9, pp. 1481-1485, Sept. 2020.

[19] X. Zhang, J. Wang, H. V. Poor, "Statistical QoS Provisioning Over Cell-Free M-MIMO-NOMA Based 5G+ Mobile Wireless Networks in the Non-Asymptotic Regime," 2020 IEEE 21st International Workshop on Signal Processing Advances in Wireless Communications (SPAWC), Atlanta, GA, USA, 2020, pp. 1-5.

[20] A. F. Molisch, M. Z. Win, "MIMO systems with antenna selection," IEEE Microwave Magazine, vol. 5, no. 1, pp. 46-56, March 2004.

[21] R. Zhang, Y. C. Liang, "Exploiting multi-antennas for opportunistic spectrum sharing in cognitive radio networks," IEEE Journal of Selected Topics in Signal Processing, vol. 2, pp. 88-102, Feb. 2008.

[22] S. Sanayei, A. Nosratinia, "Antenna selction in MIMO systems," IEEE Communications Magazine, vol. 42, pp. 68-73, Oct. 2004.

[23] A. P. Shrestha, et al., "Performance of transmit antenna selection in non-orthogonal multiple access for 5G systems," 2016 Eighth International Conference on Ubiquitous and Future Networks (ICUFN), 2016, pp. 1031-1034.

[24] X. Liu, X. Wang, "Efficient Antenna Selection and User Scheduling in 5G Massive MIMO-NOMA System," 2016 IEEE 83rd Vehicular Technology Conference (VTC Spring), 2016.

[25] F. Zhou, Z. Li, N. C. Beaulieuz, J. Cheng, Y. Wang, "Resource Allocation in Wideband Cognitive Radio with SWIPT: Max-Min Fairness Guarantees," 2016 IEEE Global Communications Conference (GLOBECOM), 2016, pp. 1-6.

[26] Q. Wu, M. Tao, D. W. K. Ng, W. Chen, R. Schober, 
"Energy-efficient resource allocation for wireless powered communication networks," IEEE Transactions on Wireless Communications, vol. 15, no. 3, pp. 2312-2327, Mar. 2016.

[27] F. Fang, H. Zhang, J. Cheng, V. C. M. Leung, "Energyefficient resource allocation for downlink non-orthogonal multiple access network," IEEE Transactions on Communications, vol. 64, no. 9, pp. 3722-3732, Sep. 2016.

[28] R. Zhang, C. K. Ho, "MIMO broadcasting for simultaneous wireless information and power transfer," IEEE Transactions on Wireless Communications, vol. 12, no. 5, pp. 1989-2001, May 2013.

[29] M. R. Amini, M. W. Baidas, "Random-Access NOMA in URLL Energy-Harvesting IoT Networks With Short Packet and Diversity Transmissions," IEEE Access, vol. 8, pp. 220734-220754, 2020.

[30] J. Zhou, Y. Sun, Q. Cao, S. Li, Z. Sun, X. Wang, ”Power Minimization for Secure Multi-User MISO NOMA System With Energy Harvesting," IEEE Transactions on Vehicular Technology, vol. 69, no. 9, pp. 10046-10058, Sept. 2020.

[31] H. Zhang, et al., "Energy Efficient Resource Management in SWIPT Enabled Heterogeneous Networks With NOMA," IEEE Transactions on Wireless Communications, vol. 19, no. 2, pp. 835-845, Feb. 2020.

[32] V. N. Vo, C. So-In, H. Tran, D. -D. Tran, T. P. Huu, "Performance Analysis of an Energy-Harvesting IoT System Using a UAV Friendly Jammer and NOMA Under Cooperative Attack," IEEE Access, vol. 8, pp. 221986222000, 2020.

[33] Z. Zhu, Z. Wang, Z. Chu, S. Huang, F. Zhou, "Maxmin fair harvested energy based beamforming designs for MISO SWIPT secrecy system," 2016 IEEE International Conference on Ubiquitous Wireless Broadband (ICUWB), 2016, pp. 1-4.

[34] D. W. K. Ng, Y. Wu, R. Schober, "Power efficient resource allocation for full-duplex radio distributed antenna networks," IEEE Transactions on Wireless Communications, vol. 15, no. 4, pp. 2896-2911, Apr. 2016.

[35] H. Wang, Z. Shi, Y. Fu, R. Song, "Outage Performance for NOMA-Aided Small Cell Networks With HARQ," IEEE Wireless Communications Letters, 2020.

[36] A. J. Muhammed, Z. Ma, Z. Zhang, P. Fan, E. G. Larsson, "Energy-Efficient Resource Allocation for NOMA Based Small Cell Networks With Wireless Backhauls," IEEE Transactions on Communications, vol. 68, no. 6, pp. 3766-3781, June 2020.

[37] S. Sobhi-Givi, M. G. Shayesteh, H. Kalbkhani, "EnergyEfficient Power Allocation and User Selection for mmWave-NOMA Transmission in M2M Communications Underlaying Cellular Heterogeneous Networks," IEEE Transactions on Vehicular Technology, 2020.

[38] N. Nguyen, T. Q. Duong, H. Q. Ngo, Z. Hadzi-Velkov, L. Shu, "Secure 5G Wireless Communications: A Joint Relay Selection and Wireless Power Transfer Approach," IEEE Access, vol. 4, pp. 3349-3359, 2016.

[39] A. J. Muhammed, Z. Ma, Z. Zhang, P. Fan, E. G. Larsson, "Energy-Efficient Resource Allocation for NOMA Based
Small Cell Networks With Wireless Backhauls," IEEE Transactions on Communications, vol. 68, no. 6, pp. 3766-3781, June 2020.

[40] M. Choi, D. Han, J. Moon, "Bi-Directional Cooperative NOMA Without Full CSIT," IEEE Transactions on Wireless Communications, vol. 17, no. 11, pp. 7515-7527, Nov. 2018.

[41] Z. Yang, Z. Ding, P. Fan, N. Al-Dhahir, "The impact of power allocation on cooperative non-orthogonal multiple access networks with SWIPT," IEEE Transactions on Wireless Communications, vol. 16, no. 7, pp. 4332-4343, Jul. 2017.

[42] A. A. Nasir, X. Zhou, S. Durrani, R. A. Kennedy, "Relaying protocols for wireless energy harvesting and information processing," IEEE Transactions on Wireless Communications, vol. 12, no. 7, pp. 3622-3636, Jul. 2013.

[43] Z. Wang, X. Yue, Z. Peng, "Full-Duplex User Relaying for NOMA System With Self-Energy Recycling," IEEE Access, vol. 6, pp. 67057-67069, 2018.

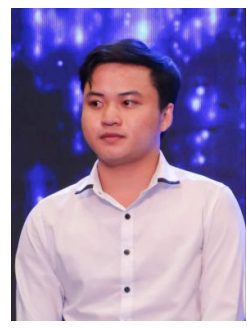

Minh-Sang Van Nguyen was born in Bentre, Vietnam. He is currently pursuing the master's degree in wireless communications. He has worked with the Industrial University of Ho Chi Minh City, Vietnam. He published more than $21 \mathrm{SCI} / \mathrm{SCIE}$ journal articles. His research interests include electronic design, signal processing in wireless communications networks, non-orthogonal multiple access, reconfigurable intelligent surfaces and physical layer security.

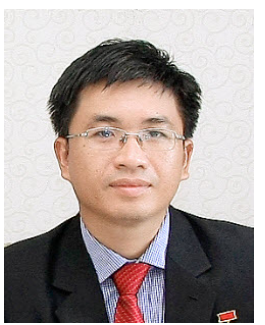

Dinh-Thuan Do (Senior Member, IEEE) received a BS, MEng, and PhD from the Vietnam National University (VNU-HCMC) in 2003, 2007, and 2013, respectively. Prior to joining The University of Texas at Austin, USA, he was an assistant professor at Asia University, Ton Duc Thang University and a senior engineer at the VinaPhone Mobile Network. Dr. Thuan is the recipient of a Golden Globe Award from the Vietnam Ministry of Science and Technology, awarded in 2015 (Top ten most excellent scientists nationwide). He also received a Creative Young Medal in 2015. He has published one book, one edited book and six book chapters. He has authored or co-authored over 100 technical papers published in peer-reviewed international journals (SCIE) and over 60 conference papers. He has presented as a Lead Guest Editor in several special issues in peer-reviewed journals. He serves as an associate editor in 5 SCIE journals. His research interests include signal processing in wireless communications networks, MIMO, NOMA, UAV networks, satellite systems, physical layer security, device-to-device transmission and energy harvesting. 


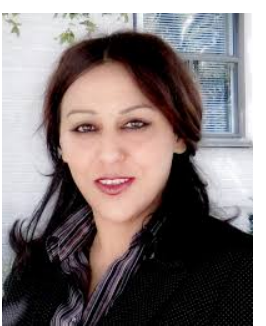

Saba Al-Rubaye received her Ph.D. degree in Electrical and Electronic Engineering from Brunel University London, United Kingdom. She is currently a Associate Professor and DRATeC Fellow. She is leading connected system research group in the School of Aerospace, Transport and Manufacturing at Cranfield University, United Kingdom. Dr AlRubaye is participating in developing industry standards by being an active voting member of IEEE P1920.2, Standard for Vehicle-to-Vehicle Communications for Unmanned Aircraft Systems and of IEEE P1932.1 standard of License/unlicensed Interoperability. She has published many papers in IEEE journals and conferences and a recipient of the best technical paper award twice published in IEEE Vehicular Technology in 2011 and 2015, respectively. She has been a general co-chair, TPC co-chair and has held other leading roles for many international conferences. She has organized and chair the 6G Network workshop in IEEEICC2020. Her main research interests include, but not limited to UAV connectivity, communication networks, artificial intelligent, safety and security of autonomous vehicle. Dr Al-Rubaye is a Chartered Engineer (CEng), member of IET, Senior member of IEEE and certified Unmanned Aircraft System (UAS) Pilot.

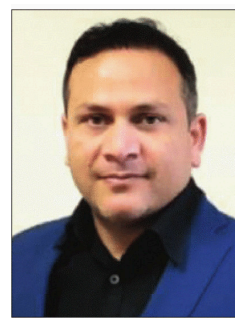

Shahid Mumtaz is a principal researcher at the Instituto de Telecomunicações, Aveiro, Portugal. He is an IET Fellow, IEEE ComSoc and ACM Distinguished Speaker, recipient of the IEEE ComSoC Young Researcher Award (2020), founder and EiC of IET Journal of Quantum Communication, ViceChair of the Europe/Africa Region-IEEE ComSoc: Green Communications \& Computing Society, and Vice-Chair of the IEEE Standard on P1932.1: Standard for Licensed/Unlicensed Spectrum Interoperability in Wireless Mobile Networks. He is the author of four technical books, 12 book chapters, 250+ technical papers $(150+$ journal/transaction, $80+$ conference), and two IEEE best paper awards in the area of mobile communications. Most of his publications are in the field of wireless communication. He is serving as a scientific expert and evaluator for various research funding agencies. He was awarded an Alain Bensoussan Fellowship in 2012. He was the recipient of the NSFC Researcher Fund for Young Scientists in 2017 from China.

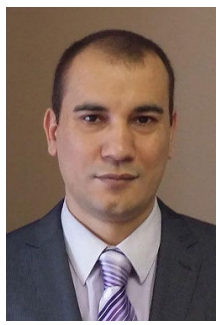

Anwer Al-Dulaimi received the $\mathrm{PhD}$ degree in electronic and computer engineering from Brunel University London, U.K., in 2012. He was a Postdoctoral Fellow with the Department of Electrical and Computer Engineering, University of Toronto, sponsored by Blackberry's advanced research team. Currently, he is a technical product owner (TPO) in the Center of Excellence at EXFO Inc., Montreal, Canada acting as the main R\&D interface supporting strategic approaches on technology and product evolutions. He is the Chair of the newly established IEEE Future Network Industry Consortium (FNIC) project working to develop a virtual testing platform for E2E network innovation. His research interests include 5G and 6G networks, cloud computing, V2X and cybersecurity. $\mathrm{He}$ is the chair of the IEEE 1932.1 Working Group "Standard for Licensed/Unlicensed Spectrum Interoperability in Wireless Mobile Network" and IEEE VTS Distinguished Lecturer. He is fellow of the British Institution of Engineering and Technology (FIET), Associate Fellow of the British higher education Academy (AFHEA) and registered as a Chartered Engineer (CEng) by the British Engineering Council since 2010. Dr. Al-Dulaimi is a member of NSERC discovery grants committee, senior member of IEEE and a voting member of IEEE MobiNet Standards Committee.

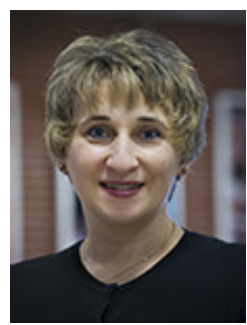

Octavia A. Dobre (M'05-SM'07-F'20) received the Dipl. Ing. and Ph.D. degrees from the Polytechnic Institute of Bucharest, Romania, in 1991 and 2000, respectively. Between 2002 and 2005, she was with New Jersey Institute of Technology, USA. In 2005, she joined Memorial University, Canada, where she is currently a Professor and Research Chair. She was a Visiting Professor with Massachusetts Institute of Technology, USA and Université de Bretagne Occidentale, France. Her research interests encompass wireless, optical and underwater communication technologies. She has (co-)authored over 350 refereed papers in these areas. Dr. Dobre serves as the Editor-in-Chief (EiC) of the IEEE Open Journal of the Communications Society. She was the EiC of the IEEE Communications Letters, Senior Editor, Editor, and Guest Editor for various prestigious journals and magazines. She also served as General Chair Technical Program Co-Chair, Tutorial Co-Chair, and Technical Co-Chair of symposia at numerous conferences. Dr. Dobre was a Fulbright Scholar, Royal Society Scholar, and Distinguished Lecturer of the IEEE Communications Society. She obtained Best Paper Awards at various conferences, including IEEE ICC, IEEE Globecom, IEEE WCNC, and IEEE PIMRC. Dr. Dobre is a Fellow of the Engineering Institute of Canada and a Fellow of the Canadian Academy of Engineering. 
2021-08-18

\title{
Exploiting impacts of antenna selection and energy harvesting for massive network connectivity
}

\author{
Van Nguyen, Minh-Sang
}

IEEE

Van Nguyen M-S, Do D-T, Al-Rubaye S, et al., (2021) Exploiting impacts of antenna selection and energy harvesting for massive network connectivity. IEEE Transactions on

Communications, Volume 69, Number 11, November 2021, pp. 7587-7602

https://doi.org/10.1109/TCOMM.2021.3106099

Downloaded from Cranfield Library Services E-Repository 